\title{
Facet evolution on supported nanostructures: Effect of finite height
}

\author{
Pak-Wing Fok, ${ }^{1,2}$ Rodolfo R. Rosales, ${ }^{3}$ and Dionisios Margetis ${ }^{4}$ \\ ${ }^{1}$ Applied and Computational Mathematics, California Institute of Technology, Pasadena, California 91125, USA \\ ${ }^{2}$ Department of Biomathematics, University of California-Los Angeles, Los Angeles, California 90095, USA \\ ${ }^{3}$ Department of Mathematics, Massachusetts Institute of Technology, Cambridge, Massachusetts 02139, USA \\ ${ }^{4}$ Department of Mathematics and Institute for Physical Science and Technology, University of Maryland, College Park, \\ Maryland 20742, USA
}

(Received 15 April 2008; revised manuscript received 30 September 2008; published 1 December 2008)

\begin{abstract}
The surface of a nanostructure relaxing on a substrate consists of a finite number of interacting steps and often involves the expansion of facets. Prior theoretical studies of facet evolution have focused on models with an infinite number of steps, which neglect edge effects caused by the presence of the substrate. By considering diffusion of adsorbed atoms (adatoms) on terraces and attachment-detachment of atoms at steps, we show that these edge or finite height effects play an important role in the structure's macroscopic evolution. We assume diffusion-limited kinetics for adatoms and a homoepitaxial substrate. Specifically, using data from step simulations and a continuum theory, we demonstrate a switch in the time behavior of geometric quantities associated with facets: the facet edge position in a straight-step system and the facet radius of an axisymmetric structure. Our analysis and numerical simulations focus on two corresponding model systems where steps repel each other through entropic and elastic dipolar interactions. The first model is a vicinal surface consisting of a finite number of straight steps; for an initially uniform step train, the slope of the surface evolves symmetrically about the centerline, i.e., the middle step when the number of steps is odd. The second model is an axisymmetric structure consisting of a finite number of circular steps; in this case, we include curvature effects which cause steps to collapse under the effect of line tension. In the first case, we show that the position of the facet edge, measured from the centerline, switches from $O\left(t^{1 / 4}\right)$ behavior to $O\left(t^{1 / 5}\right)$ (where $t$ is time). In the second case, the facet radius switches from $O\left(t^{1 / 4}\right)$ to $O(t)$. For the axisymmetric case, we also predict analytically through a continuum shock wave theory how the individual collapse times are modified by the effects of finite height under the assumption that step interactions are weak compared to the step line tension.
\end{abstract}

DOI: 10.1103/PhysRevB.78.235401

PACS number(s): 81.15.Aa, 81.10.-h, 68.55.J-

\section{INTRODUCTION}

Understanding the fundamental properties of small supported crystal mounds is important for applications in catalysis ${ }^{1}$ and nanoparticle growth. ${ }^{2}$ However, characterizing the morphology of nanoscale structures is still a challenging problem and our ability to control their evolution is fairly limited. ${ }^{3}$

As fabricated structures become smaller, finite-size effects associated with the crystal's geometry become more important. The steps that bound each monolayer in the structure are usually closed and have a finite radius of curvature. As a result, in the absence of material deposition, the perimeter of a single isolated step generally shrinks - a phenomenon that is well understood in terms of a step line tension. ${ }^{4,5}$ In addition to step curvature, another quantity that is clearly finite is the number of monolayers that make up the nanostructure. However, the effects of finite height have received much less attention in theoretical treatments compared to infinite and periodic surface features. (An infinite surface feature is one that has an infinite number of monolayers.) Furthermore, the main results to date apply only to crystals at equilibrium, where there is no facet motion. The combined effect of finite height and facet evolution remains unexplored.

For crystal surfaces undergoing relaxation below the roughening transition temperature, facets expand due to the sequential collapse of extremal steps under the influence of line tension. ${ }^{5,6}$ (An extremal step is one that has only one neighboring step.) For axisymmetric structures with a (circular) facet, scaling laws of the form $r(t)=O\left(t^{\nu}\right)$ are often observed. ${ }^{2,7-9}$ Here, $r(t)$ is the facet radius, $t$ is time, and the exponent $\nu$ depends on the initial structure's shape and the dominant transport process. ${ }^{2,10}$ However, theoretical derivations of these scaling laws ignore the effect of the substrate and are therefore valid only for infinite structures. On the other hand, most studies of supported finite structures assume an equilibrium shape under which facet motion is absent altogether. ${ }^{11-14}$

In this paper, we study the facet evolution of crystal structures relaxing toward planarity when the number of steps is finite. Our models are based on the Burton-Cabrera-Frank (BCF) theory ${ }^{15}$ for vicinal surfaces. The key microscopic processes involve adsorbed atoms ("adatoms") diffusing on terraces between steps and atoms attaching to and detaching from step edges. By balancing the flux of adatoms in and out of each step edge, one can write down equations of motion to describe the morphological evolution of the vicinal surface. Two commonly studied step geometries are the straight-step model $^{16,17}$ and the axisymmetric model ${ }^{8,9,18}$ (see Fig. 1). Our main results show that very different scaling properties arise when only a finite number of steps are present in the structure. In particular, for a set of infinitely long straight steps that are initially uniformly spaced and separate semi-infinite facets, the step positions evolve symmetrically with respect to the centerline (an assumed axis of symmetry) [see Fig. 1(a)]. The scaling with time for the position of each facet edge, measured from the centerline, switches from $O\left(t^{1 / 4}\right)$ to 

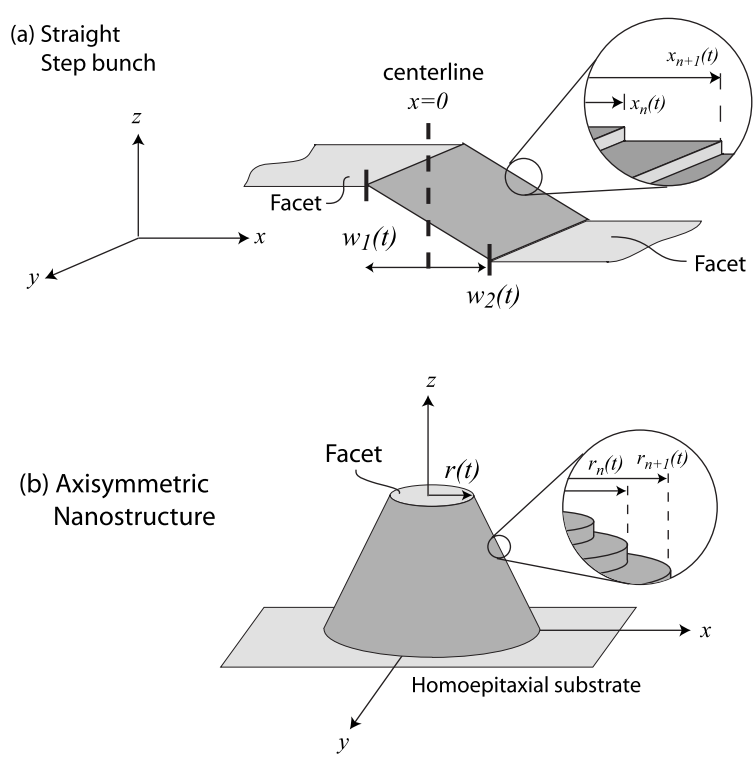

FIG. 1. The two model systems studied in this paper. (a) A finite number of straight steps separating two semi-infinite (flat) facets, possibly representing a straight-step bunch. Facet edges, $w_{1}(t)$ and $w_{2}(t)$, are measured from the centerline. (b) A finite number of circular steps, representing an axisymmetric nanostructure. A macroscopic facet, with radius $r(t)$, develops at the top of the structure as innermost steps collapse.

$O\left(t^{1 / 5}\right)$ when finite height effects become important. In the more physically relevant case of concentric circular steps surrounding a facet [see Fig. 1(b)], the analogous result is that the scaling for the facet radius switches from $r(t)$ $=O\left(t^{1 / 4}\right)$ to $O(t)$. To our knowledge, these switches in the time behavior have neither been reported nor studied before. We show these results by first numerically solving the equations of motion for a finite set of discrete steps. Then, we validate the numerical data and the scaling laws by setting up and solving a nonlinear partial differential equation (PDE) for each geometry. Interestingly, our numerical data for circular steps suggest that the ratio of the step-step interaction energy to the step line tension can be estimated by measuring the step collapse times.

When modeling the effect of the substrate, we assume that the nanostructure and its substrate are made up of the same material and that any lattice mismatch between the substrate and the crystal is small enough to be considered as negligible. Hence, the effects of mismatch-induced stress are ignored, and the base step acts as a perfect sink for all diffusing adatoms. As a result, the surface relaxation conserves volume in the axisymmetric model. Therefore the finite height effects studied in this paper are associated with geometric constraints inherent in the relaxation. Finally, the mass transport process on the structure's surface is always assumed to be terrace diffusion limited. The cases with attachment-detachment and edge-diffusion-limited kinetics, although important, complicate the analysis and lie beyond the scope of this paper.

Of the two model systems studied in this paper (see Fig. 1), the axisymmetric configuration of steps is more physically relevant because: (i) steps in experiments are usually closed and (ii) the effect of step line tension is taken into account. Although steps are never perfectly circular, there are many experiments where relaxing nanostructures are well approximated as being axisymmetric. ${ }^{5,7,19,20}$ Our results for the straight-step system should be applicable to the special situations where step line tension is unimportant. This system can perhaps be thought of as a model for a set of steps that evolves from a "step bunch" initial configuration. In this case, the width of the terraces separating the bunches is large compared to the interstep distance (inside the bunches), so that each bunch evolves in isolation at least initially.

The layout of the remainder of this paper is as follows. Section II focuses on the discrete step equations (coupled differential equations) in the straight step or one-dimensional (1D) and axisymmetric cases. For each type of geometry, we derive the governing equations of motion for a finite collection of steps and solve these equations numerically. In Sec. III, we use continuum models to find scaling laws for facet evolution in the 1D and axisymmetric settings. In particular, for the axisymmetric case, we use Lagrangian coordinates to develop a shock wave theory for facet evolution. We validate the scaling laws and the shock wave theory using numerical results from Sec. II. In Sec. IV, we conclude our paper with a discussion of our results.

\section{STEP EQUATIONS OF MOTION}

Before deriving the step equations of motion, we motivate the study of our two model systems, which are illustrated in Fig. 1. Although geometrically simple, these models capture the essential features in the time behavior of finite surface structures. We expect that results for these relatively simple models should carry over to more general geometries, at least at a qualitative level. In Fig. 1(a), the straight-step model is also worth studying in its own right because, as we will show, it has a simple analytic solution that is in exact agreement with step simulation data.

The ideas developed in the straight-step model can be used to understand the more physically relevant axisymmetric case, illustrated in Fig. 1(b). For this system, a facet develops and evolves at the top of the nanostructure as the innermost steps collapse.

We now state in more details the assumptions we make in order to set up the discrete step equations of motion for the two geometries in Fig. 1. These assumptions can be broadly divided into two categories: those that relate to the "interior steps," i.e., non-extremal steps, and those that relate to the interface between the nanostructure and the substrate or facet.

First let us consider the assumptions we make for the nanostructure itself. We assume that the surface relaxes in the absence of deposition and desorption. Furthermore, the adatom diffusion on terraces is the rate-limiting (slowest) process, with the attachment-detachment of atoms at step edges being relatively fast. We assume that no other facets develop throughout the entire course of the surface evolution.

Next, we consider the assumptions involved when modeling the effect that the substrate has on the structure's relax- 
ation. The most important assumption is that we neglect the effect of stress caused by the lattice mismatch between the supported crystal and the substrate, ${ }^{21}$ considering this to be sufficiently small. In the straight-step model [Fig. 1(a)], we set the adatom flux to be zero on both facets. In the axisymmetric case [see Fig. 1(b)], we set this flux to be zero on the substrate; this condition is equivalent to conserving the nanostructure's volume.

For the $1 \mathrm{D}$ case, we let the dimensional positions of the step edges be $\tilde{x}_{i}(\tilde{t}), i=1,2, \ldots, N$ where $\tilde{t}$ is dimensional time. We define the terrace $i$ to be the region $x_{i}<x<x_{i+1}$ between steps $i$ and $i+1$; facets are the regions $\tilde{x}_{N}<\tilde{x}<\infty$ and $-\infty<\tilde{x}<\tilde{x}_{1}$. Furthermore, the evolution of the surface preserves the symmetry of the profile about $\tilde{x}_{(N+1) / 2}$ if $N$ is odd and about $\left(\tilde{x}_{N / 2}+\widetilde{x}_{N / 2+1}\right) / 2$ if $N$ is even: the facets are always mirror images of each other.

For the axisymmetric case, we let $\widetilde{r}_{i}(\widetilde{t})$ describe the (dimensional) radius of each circular step, with the substrate being identified with the region $\widetilde{r}_{N}<\widetilde{r}<\infty$. In contrast to the straight-step case, the faceted region is not $0<\widetilde{r}<\widetilde{r}_{1}$. In fact, we will see in Sec. III B that, when the top step is about to collapse, the faceted region can be described fairly well by $0<\tilde{r}<\widetilde{r}_{2}$. [In particular, we refer the reader to Fig. 8(a) of Sec. III B for an illustration of this point.]

Broadly speaking, the facet is a macroscopic object. Its strict definition rests on the assumed behavior of the step density (positive surface slope) at the facet edge within the continuum theory, as discussed in Sec. III.

\section{A. Straight steps}

We now briefly derive the equations of motion for $N$ infinitely long straight steps using a BCF-type model. ${ }^{15}$ Consider a monotonic step train with $N$ descending steps. By mass conservation, the step velocity is

$$
\frac{d \tilde{x}_{i}}{d \tilde{t}}=\frac{\Omega}{a}\left[J_{i-1}\left(\tilde{x}_{i}\right)-J_{i}\left(\tilde{x}_{i}\right)\right],
$$

where $\Omega$ is the atomic volume, $a$ is the step height, $\tilde{x}_{i}(\widetilde{t})$ is the step position as a function of time $\tilde{t}$, and $J_{i}$ is the adatom flux defined by $J_{i}=-D \frac{\partial c_{i}}{\partial \tilde{x}}$, where $D$ is the terrace diffusivity, $\tilde{x}$ is the Cartesian coordinate, and $c_{i}(\tilde{x})$ is the adatom concentration on terrace $i$. Under the quasi-steady approximation $\left(\partial_{t} c_{i} \approx 0\right), c_{i}(\widetilde{x})$ obeys

$$
\nabla^{2} c_{i}=0
$$

which has a solution $c_{i}(\tilde{x})=A_{i} \tilde{x}+B_{i}$. The constants $A_{i}$ and $B_{i}$ are found by enforcing boundary conditions for the attachment-detachment of adatoms at step edges

$$
\begin{gathered}
\left.D \frac{\partial c_{i}}{\partial \tilde{x}}\right|_{\tilde{x}_{i}}=k\left(\left.c_{i}\right|_{\tilde{x}_{i}}-C_{i}^{\mathrm{eq}}\right), \\
-\left.D \frac{\partial c_{i}}{\partial \tilde{x}}\right|_{\tilde{x}_{i+1}}=k\left(\left.c_{i}\right|_{\tilde{x}_{i+1}}-C_{i+1}^{\mathrm{eq}}\right),
\end{gathered}
$$

where $k$ is the attachment-detachment rate of adatoms at a step edge and we have assumed the absence of an Ehrlich-
Schwoebel barrier. ${ }^{22,23}$ In Eq. (3), $C_{i}^{\text {eq }}$ is the equilibrium adatom concentration at the step edge given approximately by

$$
C_{i}^{\mathrm{eq}} \approx c_{s}\left(1+\frac{\mu_{i}}{k_{B} T}\right) .
$$

Here, $\mu_{i}$ is the step chemical potential at the $i$ th step edge, $k_{B} T$ is the Boltzmann energy, and $c_{S}$ is the equilibrium adatom concentration at an isolated straight step. The step chemical potential describes the propensity for a step edge to accept adatoms. For entropic and/or elastic dipolar interactions, $\mu_{i}$ is given by ${ }^{24}$

$$
\frac{\mu_{i}}{k_{B} T}=g\left[\left(\frac{L}{\tilde{x}_{i+1}-\tilde{x}_{i}}\right)^{3}-\left(\frac{L}{\tilde{x}_{i}-\tilde{x}_{i+1}}\right)^{3}\right],
$$

where $g$ is a dimensionless step-interaction coefficient and $L$ is the initial distance of separation between steps. Typically $L$ can range from 10 to $10^{4} \AA .^{16,25}$ We now introduce the dimensionless variables $x_{i}=\tilde{x}_{i} / L$ and $t=\tilde{t} /\left(D / k^{2}\right)$. Furthermore, we restrict ourselves to diffusion-limited kinetics so that for all $i, D / k L \ll\left(x_{i+1}-x_{i}\right)$, the dimensionless step separation. Note that the use of $k$ in our time scale, $D / k^{2}$, does not affect any dimensional results we derive since the dimensional equations of motion (and initial data) do not depend on $k$. Then, Eq. (1) with Eqs. (2)-(6) becomes

$$
\begin{aligned}
\frac{d x_{i}}{d t}= & \gamma\left[\frac{\left(\frac{1}{x_{i+2}-x_{i+1}}\right)^{3}-2\left(\frac{1}{x_{i+1}-x_{i}}\right)^{3}+\left(\frac{1}{x_{i}-x_{i-1}}\right)^{3}}{\left(x_{i+1}-x_{i}\right)}\right. \\
& \left.-\frac{\left(\frac{1}{x_{i+1}-x_{i}}\right)^{3}-2\left(\frac{1}{x_{i}-x_{i-1}}\right)^{3}+\left(\frac{1}{x_{i-1}-x_{i-2}}\right)^{3}}{\left(x_{i}-x_{i-1}\right)}\right],
\end{aligned}
$$

where

$$
\gamma=\frac{c_{s} \Omega D^{2} g}{a k^{2} L^{2}} .
$$

Equation (7) is valid only when $i=3,4, \ldots, N-3, N-2$. Steps 1, 2, N-1, and $N$ obey different equations. For example, the motion of $x_{1}$ and $x_{N}$ will be determined only by the adatom fluxes on the first and $(N-1)$ th terraces: under the quasi-steady approximation, there can be no gradients in the adatom concentration for $x<x_{1}$ and $x>x_{N}$. Hence, we set $J_{0}=J_{N}=0$, consistent with previous work. ${ }^{9}$ The step at $x_{2}$ is coupled to the steps at $x_{1}$ and $x_{3}$ through steady-state diffusion equations and their boundary conditions which involve the step chemical potentials $\mu_{1}$ and $\mu_{3}$. However, $\mu_{1}$ will only couple together $x_{1}$ and $x_{2}$ in contrast to Eq. (6). Similarly, step $N$ only experiences "one-sided" interactions with step $N-1$ and the equation for the velocity $d x_{N-1} / d t$ must be modified accordingly. Therefore, to supplement Eq. (7), we have the relations 


$$
\begin{aligned}
& \frac{d x_{1}}{d t}=\gamma\left[\frac{\left(\frac{1}{x_{3}-x_{2}}\right)^{3}-2\left(\frac{1}{x_{2}-x_{1}}\right)^{3}}{\left(x_{2}-x_{1}\right)}\right] \\
& \frac{d x_{2}}{d t}=\gamma\left[\frac{\left(\frac{1}{x_{4}-x_{3}}\right)^{3}-2\left(\frac{1}{x_{3}-x_{2}}\right)^{3}+\left(\frac{1}{x_{2}-x_{1}}\right)^{3}}{\left(x_{3}-x_{2}\right)}\right. \\
& \left.-\frac{\left(\frac{1}{x_{3}-x_{2}}\right)^{3}-2\left(\frac{1}{x_{2}-x_{1}}\right)^{3}}{\left(x_{2}-x_{1}\right)}\right] \\
& \frac{d x_{N-1}}{d t}=\gamma\left[\frac{-2\left(\frac{1}{x_{N}-x_{N-1}}\right)^{3}+\left(\frac{1}{x_{N-1}-x_{N-2}}\right)^{3}}{\left(x_{N}-x_{N-1}\right)}\right. \\
& \left.-\frac{\left(\frac{1}{x_{N}-x_{N-1}}\right)^{3}-2\left(\frac{1}{x_{N-1}-x_{N-2}}\right)^{3}+\left(\frac{1}{x_{N-2}-x_{N-3}}\right)^{3}}{\left(x_{N-1}-x_{N-2}\right)}\right] \text {, } \\
& \frac{d x_{N}}{d t}=\gamma\left[-\frac{-2\left(\frac{1}{x_{N}-x_{N-1}}\right)^{3}+\left(\frac{1}{x_{N-1}-x_{N-2}}\right)^{3}}{\left(x_{N}-x_{N-1}\right)}\right] .
\end{aligned}
$$

Equations (7) and (9)-(12), with suitable initial data, completely specify the motion of $N$ straight steps moving under the effect of entropic and/or elastic dipolar repulsions. Integrating these equations (with unit initial step separation) yields the curves in Fig. 2, where several snapshots are shown of the step density $F_{i} \equiv \frac{1}{x_{i+1}-x_{i}}$ as a function of the step position $x_{i}$. Note that at early times there is a maximum in the step density near each of the extremal steps, while far from these steps the density is still approximately constant.

Finally, note that the form of Eqs. (9)-(12) is particular to the physical situation being considered. In our case, the extremal steps are free to move outward indefinitely without restriction. This is not true in Ref. 17, for example: there, the equations of motion for steps 1,2, N-1, and $N$ are different due to step-antistep annihilations occurring at the peaks and valleys of $1 \mathrm{D}$ periodic corrugations.

\section{B. Circular steps}

The derivation of the equations of motion for the axisymmetric case is similar to Sec. II A, but is slightly modified to take into account the effect of step line tension. We use Eq. (1) but with radial positions $\widetilde{r}_{i}$ and $\tilde{r}$ replacing $\tilde{x}_{i}$ and $\widetilde{x}$. The adatom fluxes $J_{i}$ are induced by differences in the step edge

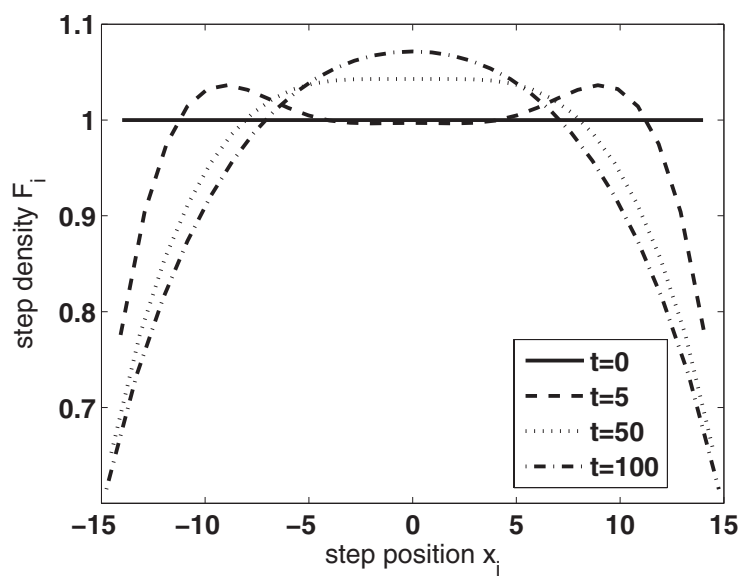

FIG. 2. Simulation data for the (discrete) straight-step density $F_{i}$ as a function of the (scaled) step position $x_{i}$. The profiles were obtained by solving Eqs. (7) and (9)-(12) numerically with the number of steps $N=31$ and the dimensionless constant [defined in Eq. (8)] $\gamma=1$.

chemical potentials $\mu_{i}$. In the axisymmetric case with diffusion-limited kinetics the $J_{i}$ take the form ${ }^{18}$

$$
J_{i}(\widetilde{r}, \widetilde{t})=-\frac{D c_{s}}{k_{B} T} \frac{1}{\widetilde{r}} \frac{\mu_{i+1}-\mu_{i}}{\ln \left(\widetilde{r}_{i+1} / \widetilde{r}_{i}\right)} .
$$

The two main differences between the straight and circularstep cases arise through the step interactions and step line tension. Both of these effects are encapsulated in the axisymmetric step chemical potential $\mu_{i}$. Instead of Eq. (6), we now have ${ }^{18}$

$$
\mu_{i}=\frac{\Omega g_{1}}{\widetilde{r}_{i}}+\frac{\Omega}{2 \pi a \widetilde{r}_{i}} \frac{\partial\left[V\left(\widetilde{r}_{i}, \widetilde{r}_{i+1}\right)+V\left(\widetilde{r}_{i-1}, \widetilde{r}_{i}\right)\right]}{\partial \widetilde{r}_{i}} .
$$

In Eq. (14), $g_{1}$ is the step line tension (a constant) and $V\left(\widetilde{r}_{i}, \widetilde{r}_{i+1}\right)$ is the interaction potential between steps $i$ and $i$ +1 . The first term, $\Omega g_{1} / \widetilde{r}_{i}$, accounts for the curvature effect of each step. Assuming entropic and/or elastic dipolar repulsions, $V$ takes the form ${ }^{6}$

$$
V\left(\widetilde{r}_{i}, \widetilde{r}_{i+1}\right)=\frac{4 \pi a^{3} g_{3}}{3} \frac{\widetilde{r}_{i} \widetilde{r}_{i+1}}{\left(\widetilde{r}_{i}+\widetilde{r}_{i+1}\right)\left(\widetilde{r}_{i+1}-\widetilde{r}_{i+1}\right)^{2}},
$$

where $g_{3}$ is the step-interaction coefficient. From Eqs. (14) and (15), we define a dimensionless parameter $g \propto g_{3} / g_{1}$ to quantify the strength of the step-step interactions relative to the step line tension. Analogous to the straight-step case, we set the adatom fluxes to be zero when $\tilde{r}<\widetilde{r}_{1}$ and $\tilde{r}>\widetilde{r}_{N}$ so that $J_{0}=J_{N}=0$. Equations (1) and (13)-(15) with suitable initial data for the step positions now completely specify the motion of all $N$ steps in the nanostructure. In dimensionless variables, the governing equations are 


$$
\begin{aligned}
& \frac{d r_{1}}{d t}=-\frac{\beta}{r_{1}}\left\{\frac{\frac{1}{r_{1}}-\frac{1}{r_{2}}+g\left[\Lambda\left(0, r_{1}, r_{2}\right)-\Lambda\left(r_{1}, r_{2}, r_{3}\right)\right]}{\ln \left(r_{2} / r_{1}\right)}\right\} \\
& \frac{d r_{2}}{d t}=-\frac{\beta}{r_{2}}\left\{\frac{\frac{1}{r_{2}}-\frac{1}{r_{3}}+g\left[\Lambda\left(r_{1}, r_{2}, r_{3}\right)-\Lambda\left(r_{2}, r_{3}, r_{4}\right)\right]}{\ln \left(r_{3} / r_{2}\right)}-\frac{\frac{1}{r_{1}}-\frac{1}{r_{2}}+g\left[\Lambda\left(0, r_{1}, r_{2}\right)-\Lambda\left(r_{1}, r_{2}, r_{3}\right)\right]}{\ln \left(r_{2} / r_{1}\right)}\right\}, \\
& \frac{d r_{i}}{d t}=-\frac{\beta}{r_{i}}\left\{\frac{\frac{1}{r_{i}}-\frac{1}{r_{i+1}}+g\left[\Lambda\left(r_{i-1}, r_{i}, r_{i+1}\right)-\Lambda\left(r_{i}, r_{i+1}, r_{i+2}\right)\right]}{\ln \left(r_{i+1} / r_{i}\right)}-\frac{\frac{1}{r_{i-1}}-\frac{1}{r_{i}}+g\left[\Lambda\left(r_{i-2}, r_{i-1}, r_{i}\right)-\Lambda\left(r_{i-1}, r_{i}, r_{i+1}\right)\right]}{\ln \left(r_{i} / r_{i-1}\right)}\right\}, \\
& i=3,4, \ldots, N-2 \text {, } \\
& \frac{d r_{N-1}}{d t}=-\frac{\beta}{r_{N-1}}\left\{\frac{\frac{1}{r_{N-1}}-\frac{1}{r_{N}}+g\left[\Lambda\left(r_{N-2}, r_{N-1}, r_{N}\right)-\Lambda\left(r_{N-1}, r_{N}, \infty\right)\right]}{\ln \left(r_{N} / r_{N-1}\right)}-\frac{\frac{1}{r_{N-2}}-\frac{1}{r_{N-1}}+g\left[\Lambda\left(r_{N-3}, r_{N-2}, r_{N-1}\right)-\Lambda\left(r_{N-2}, r_{N-1}, r_{N}\right)\right]}{\ln \left(r_{N-1} / r_{N-2}\right)}\right\}, \\
& \frac{d r_{N}}{d t}=+\frac{\beta}{r_{N}}\left\{\frac{\frac{1}{r_{N-1}}-\frac{1}{r_{N}}+g\left[\Lambda\left(r_{N-2}, r_{N-1}, r_{N}\right)-\Lambda\left(r_{N-1}, r_{N}, \infty\right)\right]}{\ln \left(r_{N} / r_{N-1}\right)}\right\}
\end{aligned}
$$

where

$$
\Lambda\left(r_{i-1}, r_{i}, r_{i+1}\right)=\frac{2 r_{i+1}}{r_{i+1}+r_{i}} \frac{1}{\left(r_{i+1}-r_{i}\right)^{3}}-\frac{2 r_{i-1}}{r_{i}+r_{i-1}} \frac{1}{\left(r_{i}-r_{i-1}\right)^{3}}+\frac{1}{r_{i}}\left[\left(\frac{r_{i+1}}{r_{i+1}+r_{i}}\right)^{2} \frac{1}{\left(r_{i+1}-r_{i}\right)^{2}}+\left(\frac{r_{i-1}}{r_{i}+r_{i-1}}\right)^{2} \frac{1}{\left(r_{i}-r_{i-1}\right)^{2}}\right] .
$$

Here $r \equiv \tilde{r} / l$ and $t \equiv \frac{\tilde{t}}{\left(D / k^{2}\right)}$, where $l$ is a length scale defined in the next paragraph and $g(l)=\frac{2}{3}(a / l)^{2}\left(g_{3} / g_{1}\right)$. Again, the use of $k$ in the chosen time scale $D / k^{2}$ does not affect any dimensional results we derive. The prefactor $\beta(l)$ $=\left(\frac{D^{2} \Omega^{2} c_{s} g_{1}}{a k^{2} k_{B} T}\right) l^{-3}$ is a dimensionless parameter that determines the time scale of evolution. One can always choose time units so that $\beta=1$ as we do in our numerical calculations.

The length scale $l$ could take one of two "natural" values. One choice is to take $l=L$, the initial step separation distance (initial terrace width), analogous to the straight-step case. This is done to simulate experiments, in which case the step positions are initialized to be $r_{n}=n, n=1,2, \ldots, N$. In our simulations, we always take $\beta(L)=1$. Another possibility is to take $l=R_{c}$, the initial radius of the base step. This second choice is made in Sec. III B on Lagrangian theory. For future reference, we note that $\beta(L) / N^{3}=\beta\left(R_{c}\right)$ and $g(L) / N^{2}=g\left(R_{c}\right)$.

Equations (16)-(21) for concentric circular steps have an important difference from Eqs. (7) and (9)-(12) for straight steps. Because of the step line tension, the radius of the top step shrinks and undergoes a monotonic collapse, resulting in a successive "peeling" of the top layer." The removal of the top layer reveals an underlying one that has a larger radius. ${ }^{5}$ Therefore, the facet evolution consists of a radial expansion along with a downward translation. This evolution has been studied in the case of infinite axisymmetric structures: in particular, for an equally spaced initial step configuration, we have $\mathrm{e}^{8,18,26} r(t)=O\left(t^{1 / 4}\right)$ and $h_{0}-h(t)=O\left(t^{1 / 4}\right)$ for $t \gg 1$, where $r(t)$ is the facet radius, $h(t)$ is the (dimensionless) vertical location of the facet relative to some reference height $h_{0}$, and $t$ is (dimensionless) time.

Another interesting quantity to study is the collapse time of the top step. These collapse times arise naturally in the solution of Eqs. (16)-(20) and can be found from experiments. ${ }^{5,7,20}$ When we integrate Eqs. (16)-(20) numerically and a collapse occurs, our convention is to remove the variable corresponding to the innermost index, $r_{j}$, for instance, and continue the integration with $r_{j+1}(t)$ as the inner- 
most step. For example, after the first collapse, $r_{1}$ is removed from the system and $r_{2}$ obeys Eq. (16) but with $r_{1} \rightarrow r_{2}, r_{2}$ $\rightarrow r_{3}$, and $r_{3} \rightarrow r_{4}$. Likewise, $r_{3}$ obeys Eq. (17) but with $r_{1}$ $\rightarrow r_{2}, r_{2} \rightarrow r_{3}, r_{3} \rightarrow r_{4}$, and $r_{4} \rightarrow r_{5}$. Hence, throughout the entire course of the integration, a particular step index always follows the same step. The behavior of these collapse times is crucial in determining the evolution (expansion) of the facet. Qualitatively, if the steps collapse frequently, the facet evolution will proceed rather quickly. On the other hand, if steps do not collapse at all, the facet will remain frozen in time. Simple scaling results have been obtained for geometrically simple structures with a practically infinite number of steps. ${ }^{8}$ For collapse times $t_{n}$, and $n \gg 1$, we have $t_{n}=O\left(n^{4}\right)$ when the spacing of steps is initially uniform (corresponding to cone shaped structures). Other initial step spacings result in different exponents. ${ }^{27}$

The scaling laws for the facet discussed above are intimately connected to the self-similarity inherent in the evolution of infinite nanostructures. . $^{26,27}$ When the kinetics is diffusion limited and the number of steps approaches infinity, future height profiles of the nanostructure look like stretched versions of past ones. This self-similarity is stable in the sense that small perturbations to an initial configuration of equally spaced circular steps eventually decay. ${ }^{27}$

In the axisymmetric case, this self-similarity property is destroyed with the onset of finite height effects. The reason for this is that the base step acts as the ultimate mass sink for the adatom flux. As a result, the radii of the last few steps grow with time as mass is expelled from the top of the structure and accumulates at the bottom. In this case, instead of self-similarity in the height profile, we show below that scaling laws in the collapse times $t_{n}$ can be determined.

In general, the collapse times depend not only on the initial values of the step radii, but on $g$ as well. Thus, for a structure initially consisting of $N$ concentric and equally spaced circular steps, let $t_{n}(N, g)$, for $n=1,2,3, \ldots$, be the collapse times. Furthermore, define the collapse time discrepancies with those of an infinite structure by

$$
E_{n}(N, g)=t_{n}(N, g)-t_{n}(\infty, g) .
$$

Figure 3(a) shows $E_{n}(N, 0.01)$ plotted as a function of $n$ for $N=40,50$, and 60. Figure 3(b) shows that the step simulation data collapse onto a single universal curve given by

$$
G\left(\frac{n}{N}, g\right)=\frac{E_{n}(N, g)}{N^{4}} .
$$

We note the following points about Eq. (23). First, $G$ is nonmonotonic. In particular, the inset of Fig. 3(b) shows the presence of a local maximum for $n / N \approx 0.45$ before the minimum at $n / N \approx 0.76$. Although not visible in the plots, the alternating pattern of minima and maxima continues for smaller values of $n / N$, with the amplitudes decreasing quickly as $n / N \rightarrow 0$. Compared to an infinite structure, the step collapse times for a finite structure occur earlier, then later, then earlier, etc. in an alternating fashion. For the data in Fig. 3(b), we found that the positions of the first four extrema (counting from the right) were at $n / N \approx 0.76,0.45$, 0.33 , and 0.25 . We do not know if extrema exist for smaller
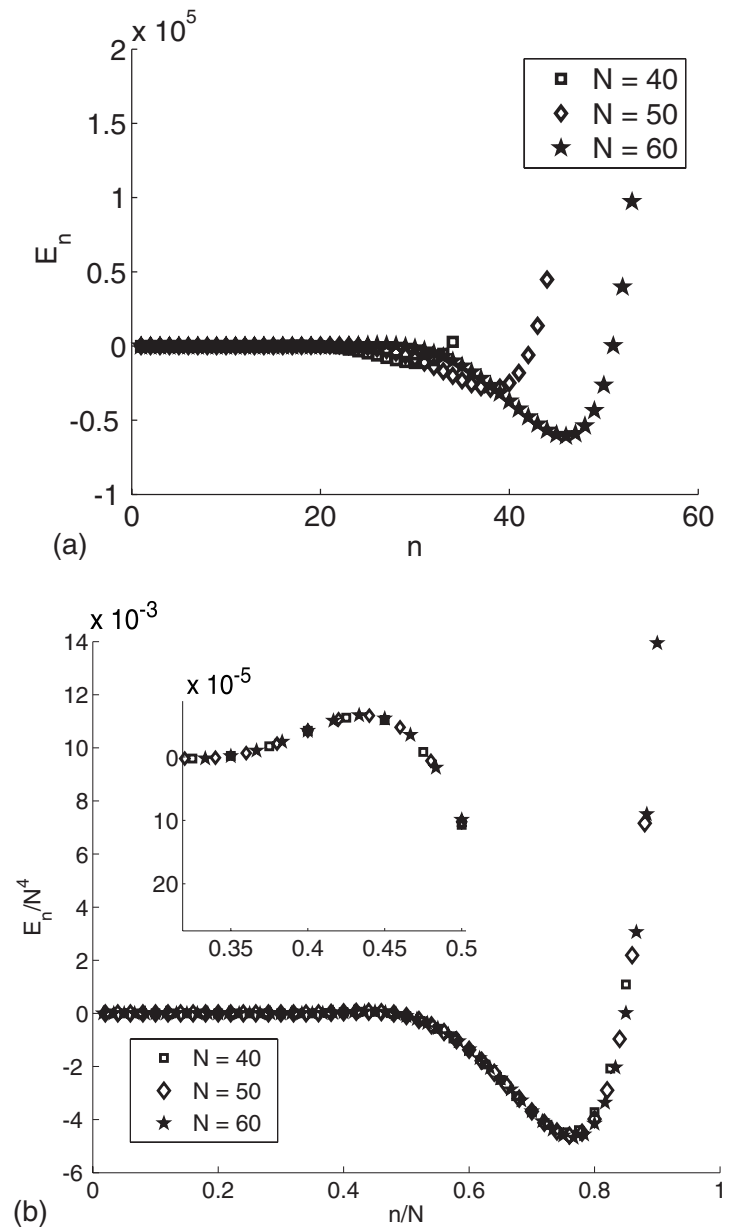

FIG. 3. (a) Circular-step (radial case) simulation data for the scaled deviations $E_{n}$ defined in Eq. (22) with the step-interaction parameter $g=0.01$. Step collapse times for an infinite structure, $t_{n}(\infty, g)$, were approximated with $t_{n}(N=700, g)$. (b) The similarity collapse data from (a) by Eq. (23). Inset shows a local maximum of $E_{n} / N^{4}$ for $n / N \approx 0.45$.

values of $n / N$. If they do, their amplitudes are too small for us to reliably detect these extrema. From the first four extrema, it appears that the spacing between them gets smaller as $n / N$ gets smaller, with the amplitudes decreasing by about an order of magnitude from one extremum to the next. Second, relation (23) seems to hold for $0.25 \leqq n / N<1$; for $n / N \leqq 0.25$, the collapse of data onto a single curve is less clear because of numerical errors. These errors are on the order of (or greater than) $|G|$, making it difficult to draw firm conclusions about the behavior of $E_{n}(N, g)$. Third, from Fig. $3(\mathrm{~b}), G(\cdot)$ appears to be a smooth continuous curve, at least when $0.25 \leqq n / N<1$. (For smaller values of $n / N$ the evidence is again inconclusive for the same reason discussed above.)

It seems reasonable to expect that a dependence such as Eq. (23) should be derivable from a continuous model in the large $N$ limit. In fact, we show that this is so in the zero- $g$ limit in Sec. III B. Remarkably, even when $N$ is as small as 40 , most of the collapse times seem to obey an underlying continuum equation. Finally, since it is known that $t_{n}(\infty, g)$ $\sim t^{*} n^{4}$ when $n \gg 1,8,26$ for large $n$ (and $N$ ) we have 


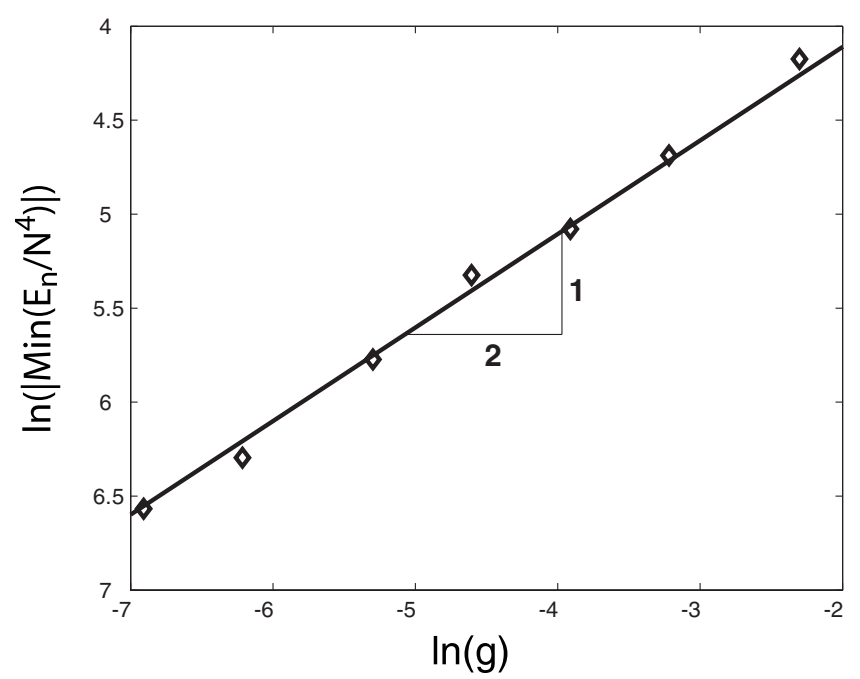

FIG. 4. Absolute minimum (in $n$ ) of scaled deviations $E_{n} / N^{4}$ from Fig. 3(b). This minimum follows the scaling law $\left|\min _{n}\left(E_{n} / N^{4}\right)\right|=O\left(g^{1 / 2}\right)$ for sufficiently small step-step interaction parameter $g$.

$t_{n}(N, g) / N^{4} \sim \widetilde{G}(n / N, g), \quad$ where $\tilde{G}(x, g) \equiv G(x, g)+t^{*}(g) x^{4}$ and $t^{*}$ has been documented for a wide range of $g$ values in Ref. 26.

With Eq. (23) established, it is natural to wonder if there is also a simple scaling of $E_{n}$ with the step-step interaction parameter $g$. Figure 4 shows that the absolute minimum of $E_{n} / N^{4}$ scales as $O\left(g^{1 / 2}\right)$ for small $g$, so that this minimum tends to zero as $g \rightarrow 0$. Again, this scaling was determined empirically: an analytic derivation is the subject of work in progress. For each value of $g$, the location of the deepest minimum in Fig. 3(b) occurred at $n / N \approx 0.76$, which is seemingly independent of $g$.

\section{CONTINUUM APPROACH}

Our goal in this section is to derive fully continuum equations that govern the relaxation of finite nanostructures for straight-step and axisymmetric geometries. These equations, in general, have nonzero solutions between two free boundaries, the positions of which correspond to the edges of facets. By solving the continuum equations, we obtain the nanostructure's slope profile and scaling laws for the facet evolution. We confirm these predictions by comparing them with results from the numerically integrated discrete step equations of Sec. II.

\section{A. One-dimensional case}

We now derive continuum equations for surface evolution following Refs. 28 and 29. First, we have the mass conservation law

$$
\frac{\partial \tilde{h}}{\partial \tilde{t}}+\Omega \nabla \cdot \mathbf{j}=0
$$

where $\tilde{h}$ is the height profile of the structure, $\Omega$ is the atomic volume, and $\mathbf{j}$ is the large-scale adatom flux. In the case of diffusion-limited kinetics, the flux $\mathbf{j}$ and the continuum surface chemical potential $\mu$ are related by ${ }^{18}$

$$
\mathbf{j}=-\frac{c_{s} D}{k_{B} T} \nabla \mu \text {. }
$$

The next step is to derive an expression for $\mu$ in terms of the slope $|\nabla \widetilde{h}|$, thus closing Eqs. (24) and (25). The resulting PDE can be solved provided that suitable boundary conditions are enforced at facet edges. The locations of these edges are unknown a priori. ${ }^{18,26,30}$ However, implementing these boundary conditions is not always straightforward. $8,26,31,32$

To derive $\mu$, we coarse grain the step chemical potential $\mu_{i}$ following the procedure used in Ref. 18. From Eq. (6) we have

$$
\frac{\mu_{i}}{k_{B} T}=g\left(F_{i}^{3}-F_{i-1}^{3}\right)
$$

with $F_{i}=\frac{1}{x_{i+1}-x_{i}}$ and $x_{i}=\tilde{x}_{i} / L$, where $L$ is the initial terrace width. This definition of $x_{i}$ is convenient since it will enable direct comparisons of PDE predictions with step simulation databased on Eqs. (7) and (9)-(12) where the same (dimensionless) $x_{i}$ is used. The quantity $F_{i}$ and the physical discrete step density $f_{i}$ are related through $F_{i}=\frac{L}{a} f_{i}$, where $f_{i}=\frac{a}{\widetilde{x}_{i+1}-\widetilde{x}_{i}}$. If we define $\Delta \tilde{x}_{i}=\tilde{x}_{i}-\tilde{x}_{i-1}=\frac{a}{f_{i-1}}$ and $\Delta x_{i}=x_{i}-x_{i-1}=1 / F_{i-1}$, then we can write

$$
F_{i}^{3}-F_{i-1}^{3}=\frac{1}{F_{i-1}} \frac{F_{i}^{3}-F_{i-1}^{3}}{\Delta x_{i}} \sim \frac{1}{F} \frac{\partial F^{3}}{\partial x} .
$$

Since $\Delta x_{i}=O(1)$, the finite difference term in Eq. (27) can only be well approximated by the derivative when the step density is a sufficiently slowly varying function of the step number $i$. This is the fundamental assumption of the continuum approach. Then, from Eq. (26), we obtain the continuous step chemical potential ${ }^{28,29}$

$$
\frac{\mu}{k_{B} T}=\frac{3 g}{2} \frac{\partial F^{2}}{\partial x} .
$$

Using Eqs. (24) and (25), we have

$$
\frac{\partial f}{\partial \widetilde{t}}+\left(\frac{\Omega c_{s} D}{k_{B} T}\right) \frac{\partial^{3} \mu}{\partial \widetilde{x}^{3}}=0,
$$

where $f=-\frac{\partial \tilde{h}}{\partial \widetilde{x}}$. Upon using Eq. (28), we have $\mathrm{e}^{17,29,33}$

$$
\frac{\partial F}{\partial t}+\frac{3 \gamma}{2} \frac{\partial^{4} F^{2}}{\partial x^{4}}=0
$$

where $x=\tilde{x} / L, t=\tilde{t} /\left(D / k^{2}\right)$, and $\gamma$ is given by Eq. (8). Equations such as Eq. (30) have often been solved using periodic

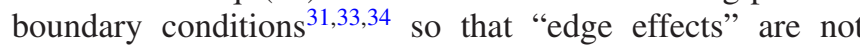
studied. These models have been used to successfully explain many experiments involving periodic profiles. ${ }^{35,36}$ Our focus in this paper is different: we would like to see how the presence of extremal steps, which only experience one-sided interactions, affects the macroscopic evolution of the vicinal surface. To this effect, we consider two model subsystems: a semi-infinite step train (the straight-step analog of the system 
studied in Ref. 8) and a (strictly) finite step train. Our aim is to find scaling laws that govern each subsystem's global behavior.

We assume that the facet edges are located at the (dimensionless) positions $w_{1}(t)$ and $w_{2}(t)$ [see Fig. 1(a)] and consider step motion under entropic and/or elastic dipolar repulsions. The governing PDE is

$$
\frac{\partial F}{\partial t}=-\alpha \frac{\partial^{4} F^{2}}{\partial x^{4}}, \quad w_{1}(t)<x<w_{2}(t),
$$

where $\alpha=\frac{3 \gamma}{2}$ and $F \equiv-\frac{\partial h}{\partial x}>0$ with $h=\tilde{h} / a$. The initial condition for Eq. (31) is

$$
F(x, 0)=F_{0}(x)>0, \quad w_{1}(0)<x<w_{2}(0),
$$

where $w_{1}(0)$ and $w_{2}(0)$ are the initial positions of the facet edges. Note that, in the numerical experiments, we took $F_{0}(x) \equiv 1$. Here, however, we set up the problem for the case of general initial conditions. Thus, the continuum formulation involves two free boundaries [the positions of which are $w_{1}(t)$ and $\left.w_{2}(t)\right]$ with a fourth-order PDE. Hence, six boundary conditions are needed, three on each facet edge, to determine the solution. We specify these conditions below.

Let us begin with the boundary conditions for the situation with a semi-infinite number of steps. In this case we take $w_{2}(t)=+\infty$ for all $t$ and assume that $F_{0}(x) \rightarrow 1$ rapidly as $x$ $\rightarrow \infty$. With only $w_{1}(t)$ unknown, Eq. (31) becomes a free boundary problem with a single free boundary ${ }^{18,26,30}$ so only five boundary conditions are needed. Let $h_{1}$ be the (constant) height of the facet to the left of $w_{1}$; hence, $h(x, t)=h_{1}$ for $x$ $\leq w_{1}$. Then the first boundary condition follows from enforcing continuity of slope ${ }^{17,18}$ across the facet edge. Note that a discontinuous slope, shown in Fig. 1, only exists at $t=0$ as a result of the initial condition. In general, for $t>0$, we expect the slope to be continuous, consistent with studies of equilibrium crystal shapes near facets. ${ }^{11,24}$ Hence, we have

$$
F\left(w_{1}\right)=0 \text {, }
$$

which is equivalent to the step density being zero at the facet edge (see Appendix A for more details on the behavior of $F$ near the facet edge). We also require $F$ to be positive to the right of $w_{1}$.

The second condition states that there is no adatom flux on the (infinitely large) facet. This fact is consistent with the adatom density being constant on the facet, satisfying the steady-state diffusion equation. Hence, $\mathbf{j}=0$ and using Eqs. (25) and (28), we have

$$
\left.\frac{\partial^{2} F^{2}}{\partial x^{2}}\right|_{x=w_{1}}=0 .
$$

The third condition comes from the continuity of the surface height and accounts for the fact that the facet height [for $\left.x<w_{1}(t)\right]$ does not change over time. Hence, by Eq. (33) we have $0=(d / d t) h\left(w_{1}(t), t\right)=\left.\partial_{t} h\right|_{x=w_{1}}=-\left.\Omega \nabla \cdot \mathbf{j}\right|_{x=w_{1}}=0$. Thus, we find

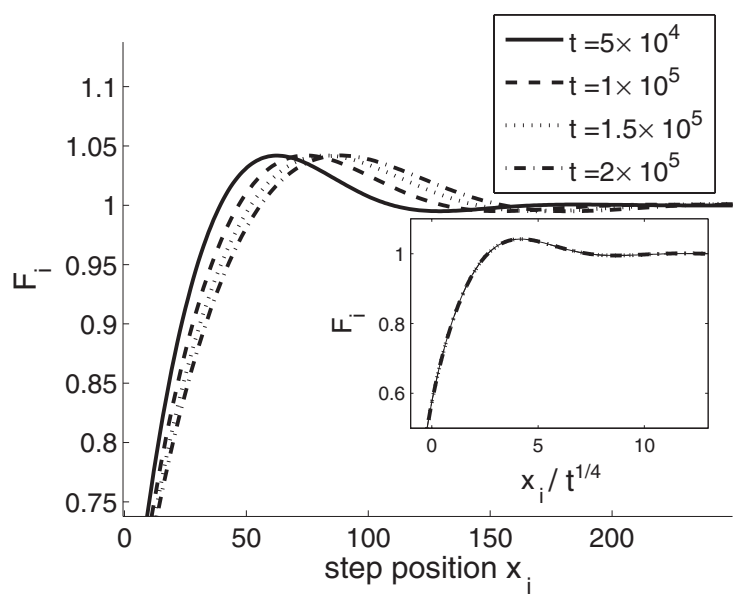

FIG. 5. Simulation data for the straight-step density obtained by integrating Eqs. (7) and (9)-(12) using a semi-infinite number of steps and the dimensionless constant $\gamma=1$ [defined in Eq. (8)]. Inset: data collapse consistent with Eq. (38).

$$
\left.\frac{\partial^{3} F^{2}}{\partial x^{3}}\right|_{x=w_{1}}=0
$$

Note that while $F^{2}$ and its derivatives (up to third order) are continuous across the facet, the derivative $\partial F / \partial x$ itself blows up as $x \rightarrow w_{1}$ (see Appendix A for more details). Finally, we impose the two far-field conditions: ${ }^{18}$

$$
\begin{gathered}
F \rightarrow 1 \quad \text { as } \quad x \rightarrow \infty, \\
\frac{\partial F}{\partial x} \rightarrow 0 \quad \text { as } \quad x \rightarrow \infty,
\end{gathered}
$$

which result from the assumption that $F_{0}(x) \rightarrow 1$ rapidly as $x \rightarrow \infty$.

In the particular case where $F_{0}(x) \equiv 1$ and $w_{1}(0)=0$, Eqs. (31)-(37) admit self-similar solutions of the form

$$
F(x, t)=f\left(x /(\alpha t)^{1 / 4}\right),
$$

with $w_{1}=w_{0} t^{1 / 4}$ for some constant $w_{0}<0$. This self-similarity is confirmed by results from step simulations in Fig. 5. The $O\left(t^{1 / 4}\right)$ scaling is a well established result for semi-infinite nanostructures. ${ }^{8,26}$ For the collapsed data shown in the inset, the step density becomes zero at a (small) negative value of the step position. Therefore, as expected, we have $w_{1}(t)$ $\sim w_{0} t^{1 / 4}$, where the constant $w_{0}<0$, consistent with a leftward facet motion.

We now turn our attention to the case of a finite structure with $N$ steps and two facets, whose edges are located at $x$ $=w_{1}(t)$ and $x=w_{2}(t)>w_{1}(t)$ [see Fig. 1(a)]. The six boundary conditions are

$$
\begin{gathered}
\frac{\partial^{3} F^{2}}{\partial x^{3}}=\frac{\partial^{2} F^{2}}{\partial x^{2}}=F=0, \\
\text { at } x=w_{1}(t) \text { and } x=w_{2}(t) .
\end{gathered}
$$

Note that Eq. (31) is a conservation law for $F$, with the flux being proportional to $\frac{\partial^{3} F^{2}}{\partial x^{3}}$. From Eq. (39) this flux vanishes at 
the facet edges; hence if $h_{1}$ and $h_{2}$ are the (constant) facet heights, then

$$
\int_{w_{1}}^{w_{2}} F(x, t) d x=h_{1}-h_{2}=N
$$

is constant as expected.

We now restrict ourselves to structures with mirror symmetry, where the solution $F=F(x, t)$ of Eq. (31) is an even function of $x$ and $w_{2}(t)=-w_{1}(t)>0$ for all times $t \geq 0$. We begin by considering similarity solutions of the form

$$
F(x, t)=\left(\frac{N^{4}}{\alpha t}\right)^{1 / 5} p(\xi), \quad \xi=\frac{x}{(N \alpha t)^{1 / 5}},
$$

where $\int_{-\infty}^{\infty} p(\xi) d \xi=1$ to satisfy Eq. (40). The substitution of ansatz (41) into Eq. (31) yields

$$
\frac{1}{5}(\xi p)^{\prime}=\left(p^{2}\right)^{\prime \prime \prime \prime} .
$$

We solve this equation assuming that $p(\xi)$ is even in $\xi$. It is therefore sufficient to consider (nonzero) solutions on the interval $\left[-\xi_{0}, 0\right]$, where $\xi_{0}=\frac{w_{1}(t)}{(N \alpha t)^{1 / 5}}$; for $\xi<-\xi_{0}, p(\xi)$ is identically zero. The boundary conditions for $p$ are $p\left(-\xi_{0}\right)=0$, $\left(p^{2}\right)^{\prime \prime}\left(-\xi_{0}\right)=0, p(0)=\lambda, p^{\prime}(0)=0$, and $(p)^{\prime \prime \prime}(0)=0$. Here, the constant $\lambda$ has been artificially introduced and is chosen to satisfy the integral constraint on $p$. Note that this constraint can be used in place of the condition $\partial^{3} F^{2} / \partial x^{3}=0$ in Eq. (39). The integral constraint ensures that $\int_{w_{1}}^{w_{2}} F d x$ is constant in time which is exactly equivalent to setting $\partial^{3} F^{2} / \partial x^{3}=0$ at the facet edges. Equation (42) was solved numerically and the solution is shown in Fig. 6. This numerical procedure (using $\lambda$ ) gives $\partial^{3} F^{2} / \partial x^{3}=0$ at the facet edges to within the accuracy of the numerical method. More details on the numerical solution of Eq. (42) are given in Appendix B.

Consider now the simple example of the initial value problem in Eqs. (31) and (32), with the boundary conditions (39), where $F_{0}(x) \equiv 1$ and $w_{2}(0)=-w_{1}(0)>0$. This, of course, yields a structure with mirror symmetry, with even $F$ and $w_{1}(t)=-w_{2}(t)$ for $t \geq 0$. For early enough times, we expect the two facets' interaction to be negligible (see Fig. 2). Hence, for these early times the solution can be well approximated by similarity solution (38) sufficiently near each of the two facets (cf. Figs. 2 and 5), yielding a $O\left(t^{1 / 4}\right)$ scaling for the facet edge position. This approximation holds for times small enough to ensure that the two maxima shown in Fig. 2 do not interact. On the other hand, the results of our numerical computations show that the solution in Eq. (41), corresponding to a $O\left(t^{1 / 5}\right)$ time behavior of the facet edge position, applies for $t$ large (see Fig. 6). Note that this second solution does not provide a good approximation for small $t$ : Fig. 6(b) shows the computed $p(\xi)$ by Eq. (41). From this plot, it is clear that the similarity solution does not obey the initial condition $F_{0} \equiv 1$.

How the transition of the facet edge position scaling from $O\left(t^{1 / 4}\right)$ to $O\left(t^{1 / 5}\right)$ occurs is not clear to us at present. This transition signifies the finite height effect. Once this effect occurs, it is easy to detect it since there are clear qualitative differences in the nanostructure's macroscopic evolution. (a)
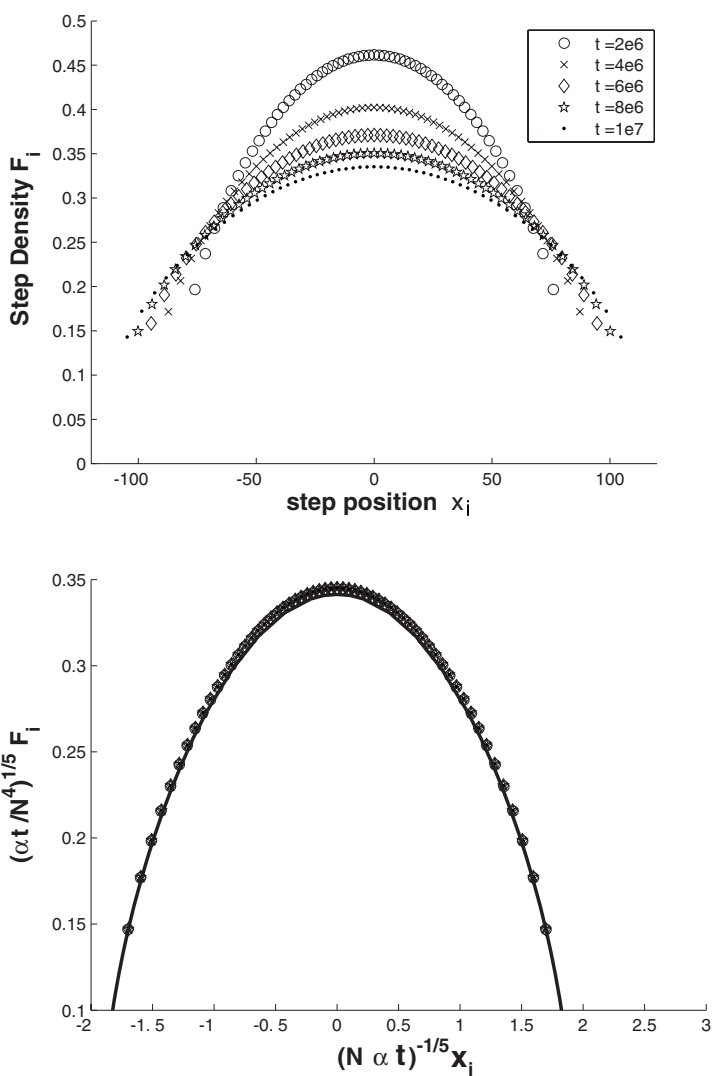

(b)

FIG. 6. (a) Simulation data for straight-step densities obtained by solving Eqs. (7) and (9)-(12) with the dimensionless constant $\gamma=1$ [see Eq. (8)] and the number of steps $N=61$. (b) Collapse of the step simulation data (symbols) and numerical solution (solid curve) of Eq. (42) with Eq. (41). The data collapse verifies the similarity solution of Eq. (41), predicting a $O\left(t^{-1 / 5}\right)$ scaling for the entire profile and a $O\left(t^{1 / 5}\right)$ scaling for the facet edge position (cf. Fig. 5).

First, the maximum step density starts to decrease in time as $O\left(t^{-1 / 5}\right)$. Second, the facet edge position switches from a $O\left(t^{1 / 4}\right)$ scaling to $O\left(t^{1 / 5}\right)$. In Fig. 2, the transition occurs roughly at $t_{0} \approx 50$. Redimensionalizing $t_{0}$, we have a transition time of

$$
\tilde{t}_{0}=t_{0} \frac{a L^{2}}{\Omega c_{s} D g}=\frac{3 t_{0}}{2}\left(\frac{L}{a}\right)^{2} \frac{a L^{3} k_{B} T}{\Omega^{2} D c_{s} g_{3}},
$$

where $D, c_{s}$, and $g_{3}$ depend on temperature and we have used $g=\frac{2 \Omega a^{2} g_{3}}{3 k_{B} T L^{3}}$ by Eq. (C6) of Appendix C.

We now discuss an application of our results to a material system. In general, it is rather difficult to obtain a full set of material parameters to evaluate our predicted transition time. However, data for the $1 \times 1$ reconstruction for $\operatorname{Si}(111)$ are available and are summarized in Appendix C. Experiments on $\mathrm{Si}(111)$ are often done at the temperature $T \approx 900{ }^{\circ} \mathrm{C}$ so that the motion of steps is fast-on the order of minutes. ${ }^{37}$ However, at this temperature, the kinetics of $\mathrm{Si}(111)$ steps is attachment-detachment limited. For our theory to be applicable, we require terrace diffusion-limited kinetics, and this 
can be achieved for $\mathrm{Si}(111)$ by reducing the temperature $T$. When $T=650 K$ and $L=100 \AA, D /(k L) \sim 0.14$ and we have a transition time of $\widetilde{t}_{0} \approx 8$ days. Although this length of time is longer than the duration of most experiments, we think that it can be greatly reduced for a material that obeys diffusion-limited kinetics at higher temperatures; for example, $\mathrm{SrTiO}_{3}(001){ }^{20}$

\section{B. Axisymmetric case}

In Sec. III A, we derived a (Eulerian) PDE for the evolution of the step density (or positive surface slope) $F(x, t)$, where $x$ is the spatial coordinate and $t$ is time. An equivalent approach to modeling nanostructure evolution is to use Lagrangian coordinates to describe the step position at a given step index and time. ${ }^{38}$ (We will describe the main advantage of using Lagrangian coordinates in the next paragraph.)

Consider Eqs. (16)-(20) with the characteristic length scale chosen to be $l=R_{c}$, the initial radius of the base step. When using Lagrangian coordinates, we replace $r_{n}(t)$, the radius of the $n$th step, by $\rho(s, t)$, where $\rho(s, t)$ is a reasonably smooth function, $s=n \delta$, and $\delta \ll 1$. For structures with $N$ steps initially, a natural choice is to set $\delta=L / R_{c}=1 / N$, treating $N$ as moderately large (say 30-100) but finite. Quantities such as $r_{n \pm 1}(t)$ are replaced by $\rho(s \pm \delta, t)=\rho(s, t) \pm \delta \cdot \rho_{s}$ $+O\left(\delta^{2}\right)$ and likewise with $r_{n \pm 2}(t)$. Using $g\left(R_{c}\right)=\delta^{2} g(L)$ and $\beta\left(R_{c}\right)=\delta^{3}$ [recall that $\beta(L)=1$ is taken in the simulations], we obtain the Lagrangian PDE (Ref. 38)

$$
\rho_{\tau}=-\frac{1}{\rho}\left\{\frac{1}{\rho}+\frac{3 g}{\rho_{s}}\left[\frac{1}{2} \frac{1}{\rho \rho_{s}}+\left(\frac{\rho \rho_{s s}}{\rho_{s}^{4}}\right)\right]\right\}_{s},
$$

where $\tau=\delta^{4} t$ and $g \equiv g(L)$. The Eulerian equivalent of Eq. (44) is also a fourth-order nonlinear PDE for the surface height and is given in Ref. 18. However, in the problem that we are concerned with, there is a good reason for using a Lagrangian formulation. The radii of the inner and outermost steps in the nanostructure are always changing. Hence, a formulation in Eulerian coordinates would involve two free boundaries whose positions are unknown a priori. In Lagrangian coordinates, the index of the base step is always fixed to be $N$ and therefore $s$ is fixed to be 1 . In contrast, the index of the first step increases monotonically as collapses occur. Therefore, the Lagrangian formulation only involves one free boundary and is simpler to work with.

Our aim here is to analytically derive the function $G(\cdot)$ that was constructed previously through numerical simulations in Fig. 3 [see Eq. (23)]. The main assumption will be that $g$, the step-interaction parameter, is small enough that we can neglect the step-interaction terms in Eq. (44). This assumption, which is somewhat analogous to considering the inviscid limit in fluid dynamics, considerably simplifies the governing Eq. (44) and allows us to obtain explicit analytic expressions for the facet radius and step collapse times. Setting $g=0$ in Eq. (44) yields

$$
\rho_{\tau}-\frac{1}{\rho^{3}} \rho_{s}=0
$$

Note that by removing step interactions completely, we have changed a fully nonlinear PDE with high-order derivatives (a)

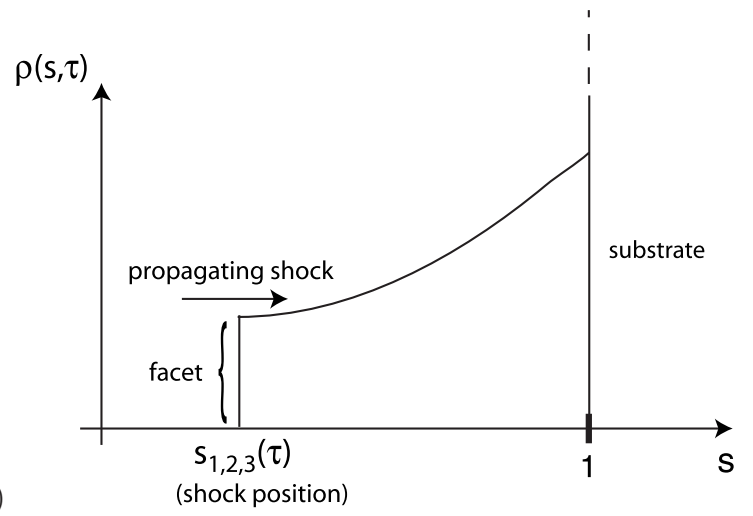

(b)



FIG. 7. (a) For an axisymmetric nanostructure, the evolving facet is represented by a propagating shock wave whose position in the $s$ (Lagrangian) coordinate is described by either $s_{1}(\tau), s_{2}(\tau)$, or $s_{3}(\tau)$, depending on whether finite height effects are significant. When $s<s_{1,2,3}(\tau)$, the step radius is $\rho=0$. (b) Characteristics (thin lines) in $(s, \tau)$ space. The position of the facet edge in Lagrangian coordinates is given by $s_{1}(\tau), s_{2}(\tau)$, and $s_{3}(\tau)$ (thick lines). Note the presence of a rarefaction fan originating at $(s, \tau)=(1,0)$, representing the effect of the motion of the base step. Characteristics also emanate from $s=1$ where the boundary conditions are unknown.

into a much simpler hyperbolic kinematic wave equation, amenable to analysis by the method of characteristics. ${ }^{39}$

One of the basic properties of hyperbolic equations is that they admit shock waves as possible solutions: there can be abrupt jumps in the solution that are mathematically represented by discontinuities. The discontinuous solution can be understood as approximating a smooth physical solution that is rapidly varying. For example, to describe fluids with low viscosity, the viscous terms in the (parabolic) Navier-Stokes equations are often dropped, giving rise to the (hyperbolic) Euler equations.

The advantage of studying a hyperbolic equation is that propagating waves and shock waves can be analyzed and understood using the method of characteristics. This method is central to the theory of hyperbolic systems ${ }^{39}$ and has been successfully applied to a diverse range of problems such as exclusion processes ${ }^{40}$ in statistical mechanics and traffic flow. ${ }^{41}$ In Fig. 7(a), the facet is represented as a sharp discontinuity in the step radius variable $\rho=\rho(s, \tau)$. As the surface relaxation proceeds, the height of the structure decreases and is accompanied by an expansion of the facet radius. This 
evolution corresponds to a rightward propagation of the shock wave and its motion is the focus of the following paragraphs.

The solution $\rho(s, \tau)$ of Eq. (45) satisfies

$$
\frac{d \rho}{d \tau}=0 \text { along } \frac{d s}{d \tau}=-\frac{1}{\rho^{3}} .
$$

The lines along which $d \rho / d \tau=0$ here are the characteristics of Eq. (45). Figure 7(b) shows the characteristic diagram corresponding to Eq. (46); note that in the presence of the substrate the variable $\rho$ can take a range of values at $(s$ $=1, \tau=0)$. This situation can be described by a set of characteristics emanating from $(1,0)$ as shown in Fig. 7(b). This set is called a "rarefaction fan," which is a concept common in the theory of PDEs (see, e.g., Refs. 39 and 41). Information from this rarefaction fan eventually affects the facet motion causing a switch in the behavior of $s(\tau)$ from $s_{1}(\tau)$ to $s_{2}(\tau)$. There is also a second switch from $s_{2}$ to $s_{3}$ due to characteristics that originate from $s=1$, the base step. However, we currently do not know what the boundary conditions at $s=1$ are, even by attempts to obtain such conditions from Eqs. (19) and (20). Therefore, we focus our attention only on the switch from $s_{1}$ to $s_{2}$, where $s_{2}(\tau)$ extends over an angle $\theta$, $0<\theta<\pi / 4$ [see Fig. 7(b)].

Note that for an infinite structure, the base step is infinitely far away and characteristics from the rarefaction fan take an infinite amount of time to reach the facet. This is consistent with $\tau=\delta^{4} t$ and taking $\delta \rightarrow 0$ : the switch from $s_{1}$ to $s_{2}$ never occurs because the scaled time in the characteristics diagram progresses infinitely slowly.

With knowledge of the functions $s_{1}$ and $s_{2}$, we can use $\tau=\delta^{4} t=t / N^{4}$ to show that the collapse times in the step simulations are given by

$$
\begin{aligned}
t_{n} & =N^{4} \sigma_{1}(n / N) \quad \text { if } \tau<\tau_{0} \\
& =N^{4} \sigma_{2}(n / N) \quad \text { if } \tau_{0}<\tau<\widetilde{\tau}_{0},
\end{aligned}
$$

where $\sigma_{1,2}$ are the inverses of $s_{1,2}$ so that $\tau=\sigma_{1,2}(s) \Leftrightarrow s$ $=s_{1,2}(\tau)$ and $s=n \delta=n / N$. Equations (47) and (48) confirm the scaling behavior observed in Fig. 3(b).

We proceed to determine the collapse times $t_{n}$. It should be clear that the volume should be conserved by the evolution. In the $g=0$ continuum limit that we consider here, this translates into the equation $\left(\rho^{2}\right)_{\tau}+(2 / \rho)_{x}=0$, which the solutions to Eq. (45) satisfy. We can then use this equation to write a "Rankine-Hugoniot" condition ${ }^{39,41}$ for the shock speed, which expresses the conservation of volume across the jump. This condition relates the facet vertical velocity, $\dot{s}_{j}(\tau)$, to the radius of the facet, $\rho\left(s_{j}, \tau\right)$, where $j=1,2$. The radius of the facet can be obtained by tracing the characteristics that intersect $s_{1}$ and $s_{2}$ in Fig. 7(b) back to the $s$ axis and the point $(1,0)$, where the initial profile $\rho(s, 0)$ is known. Hence, in principle we can find $s_{j}(\tau)$, which in turn yields the collapse times through Eqs. (47) and (48). The quantities $s_{0}$ and $\tau_{0}$ can be found from the intersection of the curves $s_{1}(\tau)$ and $s+\tau=1$. More details of the derivation can be found in Appendix D; here, we simply state our final results.

The facet radius in the presence of finite height effects is

$$
\begin{gathered}
\rho\left(s_{1}(\tau), \tau\right)=\sqrt{3} \tau^{1 / 4}, \quad \tau<1 / 9, \\
\rho\left(s_{2}(\tau), \tau\right)=B^{-1 / 3} \tau, \quad 1 / 9<\tau<\widetilde{\tau}_{0},
\end{gathered}
$$

where $B=1 / 3^{6}$. Unfortunately, our theory does not give a value for $\tilde{\tau}_{0}$ because we do not know what the boundary conditions are at $s=1$ in Fig. 7(b). Therefore, $\tilde{s}_{0}$ is also unknown.

Our prediction for the step simulation collapse times is

$$
t_{n}(N ; 0)= \begin{cases}t^{*} n^{4}, & n / N<8 / 9 \\ N^{4} \frac{\sqrt{B}}{\sqrt{1-n / N}}, & 8 / 9<n / N<\tilde{s}_{0},\end{cases}
$$

where $t^{*}=(\sqrt{3} / 2)^{12}$. The scaled deviations $E_{n}$, defined by Eq. (22), are

$$
\frac{E_{n}(N ; 0)}{N^{4}}= \begin{cases}0, & n / N<8 / 9 \\ \frac{\sqrt{B}}{\sqrt{1-n / N}}-t^{*}\left(\frac{n}{N}\right)^{4}, & 8 / 9<n / N<\widetilde{s_{0}} .\end{cases}
$$

To compare our predictions to the step simulation data, we approximate the facet radius in our simulations by the radius of the next (innermost) step when the top step collapses. Figure 8 shows a comparison of our predictions with the simulation data, assuming that $\theta$ in Fig. 7(b) is sufficiently close to $\pi / 4$ so that the $s_{2} \rightarrow s_{3}$ transition does not manifest itself. (In hindsight, this seems to be a reasonable assumption as we have never observed this second switch in our step simulations.) In Fig. 8(a) there is a switch in the behavior of the facet radius from $O\left(t^{1 / 4}\right)$ to $O(t)$. In this plot, the facet radius was approximated by measuring the radius of the innermost step $r_{n+1}\left(t_{n}\right)$ at the collapse time $t_{n}$. Note that the prediction of Eq. (49) seems to consistently underpredict the radius of the facet. Figure 8 (b) illustrates that the $t_{n}$ $=t^{*} n^{4}$ relation holds accurately for many of the early step collapses. When $\ln n \approx 4.7$, there is a switch in the behavior corresponding to characteristics from the rarefaction fan reaching the position of the facet edge $s_{2}$ in Fig. 7(b). In Fig. $8(\mathrm{c})$, we show the scaled collapse time deviations $E_{n}$ as a function of $n / N$ for different values of $g$. As $g \rightarrow 0$, the simulation results converge to the zero- $g$ solution given by Eq. (51). Note that the rapidly decaying oscillations in $E_{n}$ are not captured by the zero- $g$ solution.

Our results seem to validate the shock wave theory for facet evolution provided that $g$ is sufficiently small-but is $g$ generally small in physical situations? In Eqs. (16)-(20), the parameter $g=\frac{2}{3}(a / L)^{2}\left(g_{3} / g_{1}\right)$ depends on the initial slope of the structure which in turn depends on its method of fabrication. For example in Ref. $20, a / L \approx 0.1$ whereas in Ref. 42 , $a / L \approx 10^{-4}$. Values of $g_{1}$ and $g_{3}$ have been tabulated (see, for example, Ref. 24). For $\operatorname{Ag}(110)$ at $300 \mathrm{~K}$, we have $g_{1} a$ $=0.15 \mathrm{eV} / \AA^{2}$ and $g_{3}=0.009 \mathrm{eV} / \AA^{2}$. Taking the step height to be $a=3 \AA$, we obtain a range of values for $g \approx 3.7$ $\times 10^{-2}-3.7 \times 10^{-8}$ corresponding to $a / L \approx 10^{-1}-10^{-4}$. In general, we expect our zero- $g$ theory to be more accurate when the initial slope of the structure is smaller. 




(a)

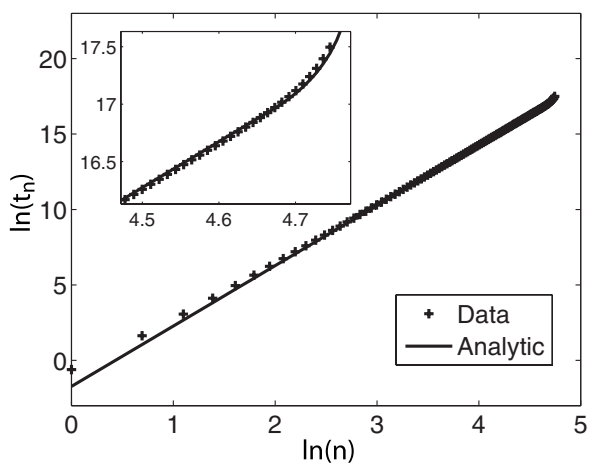

(b)

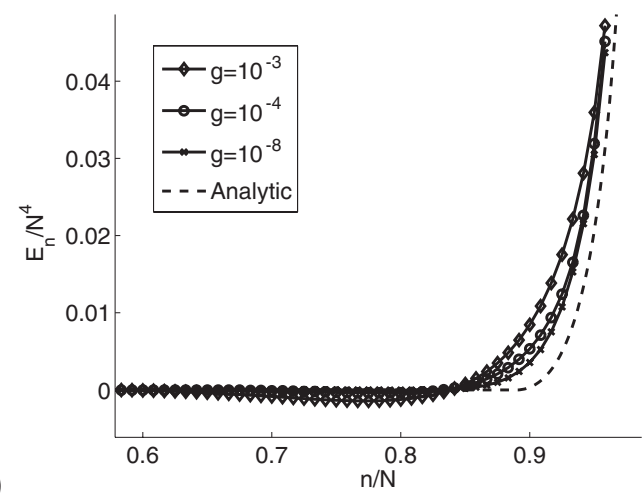

FIG. 8. Collapse time simulation data and related quantities, together with analytic predictions, for an axisymmetric nanostructure. (a) The facet radius as a function of time switches from $O\left(t^{1 / 4}\right)$ to $O(t)$, confirming prediction (49), shown as a solid line. At the collapse times $t_{n}$, the facet radius was approximated by the radius of the innermost step $r_{n+1}\left(t_{n}\right)$. The number of initial steps is $N=120$ and the step-interaction parameter is $g=10^{-8}$. (b) Collapse times from simulation data compared with theoretical prediction (50) when the initial number of steps $N=120$ and the step-step interaction parameter $g=10^{-8}$. (c) Simulation data for scaled deviations, $E_{n} / N^{4}$, from the collapse times of an infinite structure are compared to analytic prediction (51) for different values of the step-interaction parameter $g$. In the data, the collapse times for an infinite structure, $t_{n}(\infty, g)$, were approximated by $t_{n}(N=500, g)$.

Finally, let us try to compute for a specific material system the transition time, at which the facet radius switches from an $O\left(t^{1 / 4}\right)$ to an $O(t)$ behavior. Redimensionalizing $\tau_{0}$ $=1 / 9$ gives

$$
\widetilde{t}_{0}=\frac{N^{4}}{9}\left(\frac{a L^{3} k_{B} T}{\Omega^{2} D c_{s} g_{1}}\right),
$$

cf. Eq. (43). We will now calculate this transition time as before using the material parameters for $\mathrm{Si}(111)$ in Appendix C. With an initial step separation of $L=100 \AA$, we have $g$ $\equiv(2 / 3)(a / L)^{2}\left(g_{3} / g_{1}\right) \approx 4.7 \times 10^{-4}$. Hence our zero- $g$ theory should be applicable. With $N=30, T=650 \mathrm{~K}$, and the material parameters in Appendix $\mathrm{C}$, we calculate $t_{0} \approx 6$ days. As in the straight-step analysis, we expect this transition time to reduce for certain materials such as $\mathrm{SrTiO}_{3}(111)$, where the rate-limiting transport process is terrace diffusion even at temperatures as high as $1000 \mathrm{~K} .{ }^{20}$ Note that in Eq. (52), the transition time rapidly increases as $N$ increases.

\section{Comparison of boundary conditions}

We close this section with some comments about boundary conditions in the axisymmetric and straight-step cases. Consider the boundary condition for the adatom flux in the axisymmetric system [Eq. 47 of Ref. 18] and the equivalent condition for straight steps [Eq. (34)]. Both conditions specify the adatom flux at the edge of a macroscopically flat facet. In the straight-step case, this flux is zero whereas in the axisymmetric case, the adatom flux has to be nonzero to ensure that the structure's height decreases with time [viz. Eq. (24) applied at the facet edge]. The physical reason for this difference is the line tension in circular steps. Provided this line tension is sufficiently large compared to the stepstep interactions (quantified by having $g \ll 1$ ), the extremal steps of the axisymmetric and straight-step systems exhibit very different behaviors.

In our previous discussion for the axisymmetric crystal, we drew attention to the innermost (top) step which shrinks more rapidly than the other steps. ${ }^{5}$ More specifically, the top step collapses, emitting adatoms which are then absorbed by a growing second step and adjacent steps. This process gives rise to a terrace that, during most of its evolution, is much larger than the typical distance between interior steps. As previous authors pointed out, the top step in the axisymmetric system is special ${ }^{8,18,26}$ in the sense that continuum theories break down near this step and hence near the facet. The difference in the boundary conditions is essentially due to the presence of this special step in the axisymmetric case which provides a nonzero adatom flux for the facet edge. As can be seen in Fig. 6, all the terraces in the straight-step model are smoothly varying and no such special step exists. The resulting facet motion is simplified but highlights the subtleties and complications involved when modeling the evolution of actual facets that are bounded by closed steps. We emphasize again that continuum theories break down near the facet of the axisymmetric step system due to the presence of a single rapidly shrinking step on the facet. Such a step does not exist in the straight-step case and as a result, its continuum theory is valid all the way to the facet edge as can be seen in Fig. 6 .

There is another important difference between the facets in the straight-step and circular-step systems. Not only is the macroscopic adatom flux nonzero on the circular facet, but this flux also exhibits "quasiperiodic" behavior in time. A 
quasiperiodic adatom flux arises because of the regular collapse of the top step, viz. $t_{n}=O\left(n^{4}\right)$ when $n \gg 1$. Although the time between collapses is not constant, the behaviors of the facet radius and the adatom flux on the facet between two successive collapses, $\left[t_{n}, t_{n+1}\right]$ (for example), are qualitatively similar to the behaviors during $\left[t_{n+1}, t_{n+2}\right],\left[t_{n+2}, t_{n+3}\right]$, and so on. As we noted above, the facet adatom flux had been taken into account in continuum treatments of facet expansion. ${ }^{8,26}$ Furthermore the adatom flux's quasi-periodic behavior has been well documented. ${ }^{8,27}$ However, to our knowledge, the quasiperiodic time behavior has not been explicitly incorporated into any continuum theory. The solution profiles in Refs. 8 and 26 effectively rely on a "homogenized" adatom flux that results from taking a time average of the quasiperiodic one. This in turn results in solution profiles and facet boundaries that have been coarse-grained in time. More details on this coarse-graining procedure can be found in Ref. 27.

\section{CONCLUSIONS}

In this paper, we have studied the effect that the finite height has on the morphological relaxation of $1 \mathrm{D}$ and axisymmetric crystal structures. In the 1D case, we discovered that there is a switch in the time behavior of the facet edge position [measured from the centerline as shown in Fig. 1(a) and defined by where the step density extrapolates to zero in the step simulations] from $O\left(t^{1 / 4}\right)$ to $O\left(t^{1 / 5}\right)$ when finite height effects become significant. We were able to show this behavior by solving the discrete step equations numerically and from a continuum model of step motion. In the axisymmetric case, we considered the limit of weak step interactions and used a zero- $g$ theory to show that the time behavior of the facet radius switches from $O\left(t^{1 / 4}\right)$ to $O(t)$.

We were also able to quantify how step collapses are affected by the presence of the substrate [see Eq. (50)]. In infinite axisymmetric crystals, the motion of the top step is very regular and the collapse times obey a simple algebraic law, $t_{n}=O\left(n^{4}\right)$ for an initial linear cone, provided that $n$ is sufficiently large. To apply this rule in experiments, the number of steps between the facet and the substrate must be large enough for finite height effects to be negligible, i.e., the number of collapses must be much smaller than $N$, the initial number of steps. In particular, when step interactions are negligible, we have shown in this paper that the $t_{n}=O\left(n^{4}\right)$ law breaks down when $n / N>8 / 9$. We believe that our zero- $g$ theory should be applicable to many experiments where $g_{3} / g_{1}$ is moderate and $a / L \ll 1$, rendering the key material parameter (relative step-step interaction strength) $g$ small, $g \ll 1$.

Although we think our theory will be quite accurate when $g$ is negligible, it is important to understand how a nonzero $g$ will affect our predictions. We expect that the most important difference between experimental data and our zero- $g$ theory will be that collapse times are affected much more quickly by finite height effects. Recall that the zero- $g$ theory predicts nonzero deviations, $\left|E_{n}\right| / N^{4}>0$, from the $N=\infty$ case only when $n / N>8 / 9$. For nonzero $g$, the simulation data suggest that these deviations become nonzero for $n / N<8 / 9$. How- ever, these effects may not be experimentally detectable for small values of $n / N$. Furthermore, the zero- $g$ theory does not predict the nonmonotonic behavior of $G$ : a full solution to Eq. (44) should yield curves for $G$ that are: (i) nonzero for $n / N<8 / 9$ and (ii) nonmonotonic. We leave the task of deriving these curves for future work.

When we studied axisymmetric structures in this section, the focus was on step configurations that were uniformly spaced initially. However, we think that the long-time $O(t)$ behavior of the facet radius is actually independent of the initial shape. Note that in Fig. $7(\mathrm{~b}), s_{1}(\tau)$ is determined by the initial condition. In contrast, $s_{2}(\tau)$ is determined by the rarefaction fan. We expect that in the $g \rightarrow 0$ limit, changing the initial condition will modify the evolution of the facet radius for $\tau<\tau_{0}$, but the motion of the facet for $\tau_{0}<\tau<\widetilde{\tau}_{0}$ will remain unaffected, apart from a change in the $B^{-1 / 3}$ prefactor in Eq. (49) coming from a modified matching condition.

The result $\min _{n}\left(E_{n} / N^{4}\right)=O\left(g^{1 / 2}\right)$ demonstrated in Fig. 4 raises the prospect of inferring the ratio $g_{3} / g_{1}$ simply by measuring step collapse times provided that the ratio $a / L(L$ is the initial terrace width) and the initial number of steps $N$ are known. Constructing the $E_{n}$ requires knowledge of collapse times for infinite structures, which can come from the theoretical result $t_{n}=O\left(n^{4}\right)$. A full solution to Eq. (44) should also confirm the $O\left(g^{1 / 2}\right)$ behavior which was obtained in this paper solely from numerical simulations.

The main extensions of this work relate to boundary conditions for the base step. As pointed out in Sec. III B, we were not able to derive boundary conditions for the base step from Eqs. (19) and (20). This resulted in our shock wave theory being somewhat incomplete. In particular, we did not know the boundary conditions at $s=1$ in Fig. 7(b) and were not able to find $s_{3}(\tau)$. On a related issue, the case of heteroepitaxial substrates was not considered at all in this paper. Although strain effects have been studied by many researchers in the context of epitaxial growth (see, for example, Ref. 43) it is unclear how the presence of a lattice mismatch would affect the motion of the base step and the facet in our present system. Finally, we mentioned in Sec. III C the issue of a quasiperiodic adatom flux on the facet. An attempt was made to account for this flux in Ref. 27 but an implementation of this condition is still lacking.

\section{ACKNOWLEDGMENTS}

The authors thank R. V. Kohn and E. D. Williams for valuable discussions. R.R.R. was partially supported by NSF-DMS under Contract No. 0813648. D.M. was supported in part by NSF-MRSEC under Contract No. DMR0520471 at the University of Maryland and also appreciates the support of the Maryland NanoCenter.

\section{APPENDIX A: EXPANSION OF EQ. (31) NEAR FACET}

We seek a solution for $F$ near the facet $w_{1}$ in the form $F(x, t)=\sum_{i=1}^{\infty} A_{n}(t)\left(x-w_{1}(t)\right)^{n / 2}$, which satisfies condition (33), with $A_{1}(t)>0$; similar expansions were used in Refs. 8 and 
26 to describe the facet evolution in axisymmetric structures. Then conditions (34) and (35) imply that

$$
\begin{gathered}
A_{1} A_{2}=0, \\
2 A_{1} A_{3}+A_{2}^{2}=0, \\
A_{2} A_{3}+A_{1} A_{4}=0, \\
2 A_{2} A_{4}+2 A_{1} A_{5}+A_{3}^{2}=0,
\end{gathered}
$$

so that $A_{2}=A_{3}=A_{4}=A_{5}=0$. By substituting

$$
F=A_{1}\left(x-w_{1}\right)^{1 / 2}+A_{6}\left(x-w_{1}\right)^{3}+\cdots+A_{n}\left(x-w_{1}\right)^{n / 2}+\cdots
$$

directly into Eq. (31), we can obtain all the $A_{n}, n \geq 6$ in terms of $A_{1}$ and $w_{1}$. For example, by setting equal coefficients of $\left(x-w_{1}\right)^{-1 / 2}$ we obtain $A_{6}=4 \dot{w}_{1} / 105 \alpha$. Thus, two free coefficients (which are functions of time), $A_{1}(t)$ and $w_{1}(t)$, are left to be determined by conditions (36) and (37).

\section{APPENDIX B: NUMERICAL SOLUTION OF EQ. (42)}

First, we map the original domain $\left[-\xi_{0}, 0\right]$ onto $[-1,0]$ via the change in variable $y=\xi / \xi_{0}$. Second, the dependent variable is taken to be $q \equiv p^{2}$ to regularize the solution near the facet edge: it can be shown that $p(\xi)=O\left(\sqrt{\xi-\xi_{0}}\right)$ and, thus, $q(\xi)=O\left(\xi-\xi_{0}\right)$ as $\xi \rightarrow \xi_{0}$. Singularities at facet edges are due to the nonanalyticity of the surface free-energy density. ${ }^{28,29,44}$ Equation (42) becomes

$$
q^{\prime \prime \prime \prime}-\frac{\xi_{0}^{4}}{5}\left(\frac{y q^{\prime}}{2 \sqrt{q}}+\sqrt{q}\right)=0,
$$

which is to be solved for $q(y)$ on $[-1,0]$ using the boundary conditions

$$
\begin{aligned}
& q(-1)=0, \\
& q^{\prime \prime}(-1)=0, \\
& q(0)=\lambda^{2}, \\
& q^{\prime}(0)=0, \\
& q^{\prime \prime \prime}(0)=0 .
\end{aligned}
$$

Our method involves solving Eqs. (B1)-(B6) to obtain $p$ $=\sqrt{q}$ for some $\lambda$ and then using a root-finding procedure to find the unique $\lambda$ that yields $\int_{-\xi_{0}}^{0} p(\xi) d \xi=1 / 2$. As we show below, this method yields a solution for $p$ consistent with condition (35), which is not invoked explicitly in Eqs. (B2)-(B6).

Equation (B1) is singular at $y=-1$. The numerical domain of solution is therefore restricted to be $[-1+d, 0]$ for some $0<d \ll 1$. Our implementation used $d=1 / 100$. Boundary conditions (B2) and (B3) must be replaced by values of $q$ and $q^{\prime \prime}$ at $y=-1+d$, obtained through a series expansion. We take $q(y)=\sum_{m=0}^{\nu} c_{m}(y+1)^{m / 2}$ to be the form of $q$ near the facet edge. ${ }^{17}$ (The coefficients $c_{m}$ here should not be confused with the terrace adatom densities, $c_{i}$, used in the main text.) In our numerical procedure, we used $\nu=12$ and this value gave sufficient accuracy in order to compare with step simulation data. Noting Eq. (B3), it is straightforward to show that $c_{1}$ $=c_{3}=c_{4}=0$ in the expansion for the variable $q$. Substituting the series expansion for $q$ into Eq. (B1), we obtain

$$
\begin{gathered}
c_{5}=0, \quad c_{7}=-\frac{8}{525} \sqrt{c_{2}} \xi_{0}^{4}, \\
c_{8}=0, \quad c_{9}=\frac{8}{1575} \sqrt{c_{2}} \xi_{0}^{4}, \\
c_{10}=0, \quad c_{11}=-\frac{4}{3465} \frac{c_{6}}{\sqrt{c_{2}}} \xi_{0}^{4}, \quad c_{12}=-\frac{1}{1200} \frac{c_{7}}{\sqrt{c_{2}}} \xi_{0}^{4} .
\end{gathered}
$$

Therefore, the coefficients $c_{m}, m=5,6, \ldots, 12$ can be obtained in terms of $\left(c_{2}, c_{6}\right)$. These two constants are introduced as additional (unknown) parameters along with $\xi_{0}$. Since Eq. (B1) is of fourth order, we require seven boundary conditions altogether. The three conditions (B4)-(B6) apply at $y=0$ and the remaining four conditions are given by series expansions at $-1+d$ :

$$
\begin{gathered}
q(-1+d)=c_{2} d+c_{6} d^{3}+O\left(d^{7 / 2}\right), \\
q^{\prime}(-1+d)=c_{2}+3 c_{6} d^{2}+\frac{7}{2} c_{7} d^{5 / 2}+O\left(d^{7 / 2}\right), \\
q^{\prime \prime}(-1+d)=6 c_{6} d+\frac{35}{4} c_{7} d^{3 / 2}+\frac{63}{4} c_{9} d^{5 / 2}+O\left(d^{7 / 2}\right), \\
q^{\prime \prime \prime}(-1+d)=6 c_{6}+\frac{105}{8} c_{7} d^{1 / 2}+\frac{315}{8} c_{9} d^{3 / 2}+\frac{693}{8} c_{11} d^{5 / 2} \\
+120 c_{12} d^{3}+O\left(d^{7 / 2}\right) .
\end{gathered}
$$

The numerical solution was obtained using MATLAB's boundary-value problem solver BVP4C. After recovering $p(\xi)$ from $q(y)$, the area under $p$ was found using a trapezoidal rule for each value of $\lambda$. Numerically, we found that $\left(c_{2}, c_{6}, \xi_{0}, \lambda\right)=(0.175,0.000,1.936,0.341)$ gave a $p(\xi)$ that satisfied $\int_{-\xi_{0}}^{0} p(\xi) d \xi=1 / 2$. This solution is plotted in Fig. 6(b). Note that $c_{6}=0$ within the accuracy of our algorithm; hence, there is no cubic term in Eq. (B8), and the behavior of $q(-1+d)$ is consistent with the form of expansion (A5) as expected.

\section{APPENDIX C: MATERIAL PARAMETERS}

Here we give the values for key material parameters of $\mathrm{Si}(111)$ and derive the step energetic parameters $g_{1}$ and $g_{3}$ used in Eqs. (52) and (43), respectively.

The terrace diffusivity and attachment-detachment coefficient take the form ${ }^{24}$ 
TABLE I. Table of material parameters for $\mathrm{Si}(111)$ used to calculate key constants in step and continuum models. $T=900{ }^{\circ} \mathrm{C}$ for the values of $D^{*}$ and $k^{*}$.

\begin{tabular}{lccc}
\hline \hline Parameter & Parameter name & Value & Reference \\
\hline$D^{*}$ & Terrace diffusivity & $3.4 \times 10^{10} \AA^{2} / \mathrm{s}$ & Ref. 37, Table I \\
$k^{*}$ & Attachment-detachment coefficient & $6.9 \times 10^{6} \AA / \mathrm{s}$ & Ref. 37, Table I \\
$\epsilon$ & Kink energy & $0.202 \mathrm{eV}$ & Ref. 24, Table III \\
$E_{k}$ & Energy barrier for attachment-detachment & $0.61 \mathrm{eV}$ & Ref. 24, Table VIII \\
$E_{d}$ & Energy barrier for diffusion & $0.97 \mathrm{eV}$ & Ref. 24, Table VIII \\
$A$ & Step elastic interaction potential coefficient & $0.16 \mathrm{eV} \AA$ & Ref. 45 \\
$a$ & Step height & $3.1 \AA$ & Ref. 2 \\
\hline \hline
\end{tabular}

$$
\begin{aligned}
& D=D_{0} e^{-E_{d} / k_{B} T}, \\
& k=\frac{k_{0}}{c_{\mathrm{eq}}} e^{-E_{k} / k_{B} T},
\end{aligned}
$$

where we take $c_{\mathrm{eq}}=a^{-2} e^{-2 \epsilon / k_{B} T}$ and the prefactors $k_{0}$ and $D_{0}$ are determined from $D^{*}$ and $k^{*}$, which apply at $900{ }^{\circ} \mathrm{C}$ in Table I.

The step line tension $g_{1}$ is determined by ${ }^{24}$

$$
g_{1}=\frac{\beta}{a}
$$

where $\beta=\frac{\epsilon}{a}-\frac{k_{B} T}{a} \ln \operatorname{coth}\left(\frac{\epsilon}{2 k_{B} T}\right)$ is the step-free energy. ${ }^{24}$

In general, the step-step interaction energy $g_{3}$ accounts for both entropic and elastic repulsions. For purely entropic interactions, $g_{3}$ can be expressed in terms of the step stiffness through $g_{3}=\frac{(\pi k T)^{2}}{6 q^{3} \beta} \cdot{ }^{24}$ If elastic dipolar repulsions with potential $U(x)=A / x^{2}$ are also included [where $A$ for $\operatorname{Si}(111)$ is given in Table I], this expression is modified to ${ }^{24}$

$$
g_{3}=\frac{\left(\pi k_{B} T\right)^{2}}{24 a^{3} \beta}\left(1+\sqrt{1+\frac{4 A \beta}{\left(k_{B} T\right)^{2}}}\right)^{2} .
$$

Finally, the dimensionless step-step interaction parameter $g$ in Eq. (6) can be found in terms of $g_{3}$ by taking the limit $\widetilde{r}_{i-1}, \widetilde{r}_{i}, \widetilde{r}_{i+1} \rightarrow \infty$ while keeping $\left(\widetilde{r}_{i+1}-\widetilde{r}_{i}\right)$ fixed in Eqs. (14) and (15). This procedure yields

$$
\mu_{i} \sim\left(\frac{2 \Omega a^{2} g_{3}}{3 L^{3}}\right)\left[\frac{L^{3}}{\left(\widetilde{r}_{i}-\widetilde{r}_{i-1}\right)^{3}}-\frac{L^{3}}{\left(\widetilde{r}_{i+1}-\widetilde{r}_{i}\right)^{3}}\right] .
$$

Hence, by comparison with Eq. (6), we obtain

$$
g=\frac{2 \Omega a^{2} g_{3}}{3 k_{B} T L^{3}},
$$

which can be calculated using the values in Table I. The atomic volume is calculated as $\Omega=a^{3}$.

\section{APPENDIX D: DERIVATION OF FACET RADIUS EVOLUTION AND ASSOCIATED COLLAPSE TIMES}

When considering shocks in the solutions of Eq. (45), a conservation form $u_{\tau}+\phi_{s}=0$ is needed. Here $u$ is the density of some conserved quantity (conserved even across shocks) and $\phi$ is its flux. Then, the Rankine-Hugoniot (RH) jump conditions $^{39}$ apply and provide an equation for the shock velocity $\dot{s}$, namely, $\dot{s}=[\phi] /[u]$. Here the square brackets indicate the jump in the enclosed quantity across the shock discontinuity. For example, $[u]=u_{+}-u_{-}$, with $u_{ \pm}$being the values of $u$ immediately to the right (+) and left (-) of the shock position $s=s(\tau)$.

In the axisymmetric system, volume is conserved, with the density given by the step area $u=\pi \rho^{2}$. Thus the appropriate conservation form for Eq. (45) is

$$
\left(\rho^{2}\right)_{\tau}+\left(\frac{2}{\rho}\right)_{s}=0,
$$

taking $u=\rho^{2}$ and $\phi=2 / \rho$. When the facet is treated as a shock, a further subtlety arises, as Eq. (D1) does not supply a value for the volume flux to the left of the facet edge in Fig. 7(a) where $\rho=\rho_{-}=0$. However, to the left of the shock, no steps are present and clearly there can be no contribution to the volume flux. Therefore, a reasonable assumption is to set this flux to zero $\phi_{-}=0$.

Thus, from the RH shock conditions corresponding to Eq. (D1), we have the equation

$$
\frac{d s_{j}}{d \tau}=\frac{[2 / \rho]}{\left[\rho^{2}\right]}=\frac{2}{\rho_{+}^{3}}, \quad \text { for } j=1 \text { and } 2,
$$

for the shock speed, where $\rho_{+}$is, in fact, the facet radius. Then using the characteristic Eq. (46), we obtain the implicit solution valid when $s>s_{1}(\tau), \tau>0$, and $s+\tau<1$ :

$$
\begin{gathered}
\rho=s+\tau / \rho^{3} \\
\Rightarrow \rho(s, \tau)=\tau^{1 / 4} R\left(s / \tau^{1 / 4}\right),
\end{gathered}
$$

where the function $R$ satisfies

$$
R(z)=z+\frac{1}{R^{3}(z)} .
$$

Using Eq. (D2), $s_{1}(\tau)$ must satisfy the ordinary differential equation 


$$
\dot{s}_{1}(\tau)=\frac{2}{\tau^{3 / 4} R^{3}\left(\frac{s_{1}(\tau)}{\tau^{1 / 4}}\right)} .
$$

We seek a solution to this equation [subject to $s_{1}(0)=0$ ] in the form $s_{1}(\tau)=c \tau^{1 / 4}$ for some constant $c$. Substituting into Eq. (D6), we obtain $c R^{3}(c)=8$ and using Eq. (D5), we obtain $c=\left(\frac{2}{\sqrt{3}}\right)^{3}$. Therefore the facet radius is

$$
\rho\left(s_{1}(\tau), \tau\right)=R(c) \tau^{1 / 4}=\sqrt{3} \tau^{1 / 4}
$$

when $\tau<\tau_{0}$, in agreement with Ref. 18. The step simulation collapse times are

$$
t_{n}=\frac{1}{\delta^{4}} \frac{s_{1}^{4}}{c^{4}}=t^{*} n^{4},
$$

where $t^{*}=c^{-4}=(\sqrt{3} / 2)^{12}$. Values of $t^{*}$ when $g \neq 0$ were established numerically in Ref. 26 along with its asymptotic behavior when $g \rightarrow 0$. The value of $s$ at which $s_{1}(\tau)$ switches to $s_{2}(\tau)$ satisfies

$$
\begin{aligned}
& 1-s=t^{*} s^{4} \\
& \Rightarrow s_{0}=8 / 9 \\
& \Rightarrow \tau_{0}=1 / 9 .
\end{aligned}
$$

The characteristics originating from $(\tau, s)=(0,1)$ satisfy

$$
\begin{gathered}
\tau=\alpha(1-s) \\
\Rightarrow \frac{d s}{d \tau}=-\frac{1}{\alpha}=-\frac{1}{\rho^{3}},
\end{gathered}
$$

where $1<\alpha<\tan \left(\frac{\pi}{4}+\theta\right)$. Therefore,

$$
\rho(s, \tau)=\left(\frac{\tau}{1-s}\right)^{1 / 3},
$$

when $1<\tau /(1-s)<\tan \left(\frac{\pi}{4}+\theta\right)$. Using Eq. (D2) with Eq. (D14), we obtain

$$
\begin{aligned}
\dot{s}_{2}(\tau) & =\frac{2\left(1-s_{2}\right)}{\tau} \\
\Rightarrow s_{2}(\tau) & =1-\frac{B}{\tau^{2}},
\end{aligned}
$$

for some constant $B$ which is found by the matching condition $s_{1}\left(\tau_{0}\right)=s_{2}\left(\tau_{0}\right)$ :

$$
B=\left(1-s_{0}\right) t^{* 2} s_{0}^{8}=1 / 3^{6} .
$$

Note that from Eq. (D14), we have a switch in the time behavior of the facet radius

$$
\begin{gathered}
\rho\left(s_{1}(\tau), \tau\right)=\sqrt{3} \tau^{1 / 4}, \quad \tau<\tau_{0}, \\
\rho\left(s_{2}(\tau), \tau\right)=B^{-1 / 3} \tau, \quad \tau_{0}<\tau<\widetilde{\tau}_{0} .
\end{gathered}
$$

From Eq. (D16), we have

$$
\tau=\frac{\sqrt{B}}{\sqrt{1-s_{2}}},
$$

where $\sqrt{B}=3^{-3}$. Therefore, our predictions for the step collapse times $t_{n}$ and scaled deviations $E_{n} / N^{4}$ are

$$
t_{n}(N ; 0)= \begin{cases}t^{*} n^{4}, & n / N<8 / 9 \\ N^{4} \frac{\sqrt{B}}{\sqrt{1-n / N}}, & 8 / 9<n / N<\tilde{s}_{0},\end{cases}
$$

$$
\frac{E_{n}(N ; 0)}{N^{4}}= \begin{cases}0, & n / N<8 / 9 \\ \frac{\sqrt{B}}{\sqrt{1-n / N}}-t^{*}\left(\frac{n}{N}\right)^{4}, & 8 / 9<n / N<\widetilde{s}_{0}\end{cases}
$$

${ }^{1}$ Catalysis and Electrocatalysis at Nanoparticle Surfaces, edited by A. Wieckowski, E. R. Savinova, and C. G. Vayenas (Dekker, New York, 2003).

${ }^{2}$ A. Ichimiya, K. Hayashi, E. D. Williams, T. L. Einstein, M. Uwaha, and K. Watanabe, Phys. Rev. Lett. 84, 3662 (2000).

${ }^{3}$ C. R. Henry, Prog. Surf. Sci. 80, 92 (2005).

${ }^{4}$ J. G. McLean, B. Krishnamachari, D. R. Peale, E. Chason, J. P. Sethna, and B. H. Cooper, Phys. Rev. B 55, 1811 (1997).

${ }^{5}$ K. Thürmer, J. E. Reutt-Robey, E. D. Williams, M. Uwaha, A. Emundts, and H. P. Bonzel, Phys. Rev. Lett. 87, 186102 (2001).

${ }^{6}$ S. Tanaka, N. C. Bartelt, C. C. Umbach, R. M. Tromp, and J. M. Blakeley, Phys. Rev. Lett. 78, 3342 (1997).

${ }^{7}$ D. B. Dougherty, K. Thürmer, M. Degawa, W. G. Cullen, J. E. Reutt-Robey, and E. D. Williams, Surf. Sci. 554, 233 (2004).

${ }^{8}$ N. Israeli and D. Kandel, Phys. Rev. B 60, 5946 (1999).
${ }^{9}$ M. Uwaha and K. Watanabe, J. Phys. Soc. Jpn. 69, 497 (2000).

${ }^{10}$ M. Uwaha, J. Phys. Soc. Jpn. 57, 1681 (1988).

${ }^{11}$ M. Degawa, F. Szalma, and E. D. Williams, Surf. Sci. 583, 126 (2005).

${ }^{12}$ M. Degawa and E. D. Williams, Surf. Sci. 595, 87 (2005).

${ }^{13}$ P. Müller and R. Kern, Surf. Sci. 457, 229 (2000).

${ }^{14}$ H. P. Bonzel, Phys. Rep. 385, 1 (2003).

${ }^{15}$ W. K. Burton, N. Cabrera, and F. C. Frank, Philos. Trans. R. Soc. London, Ser. A 243, 299 (1951).

${ }^{16}$ W. Hong, H. N. Lee, M. Yoon, H. M. Christen, D. H. Lowndes, Z. Suo, and Z. Zhang, Phys. Rev. Lett. 95, 095501 (2005).

${ }^{17}$ N. Israeli and D. Kandel, Phys. Rev. B 62, 13707 (2000).

${ }^{18}$ D. Margetis, M. J. Aziz, and H. A. Stone, Phys. Rev. B 71, 165432 (2005).

${ }^{19}$ Y. Homma, H. Hibino, T. Ogino, and N. Aizawa, Phys. Rev. B 
58, 13146 (1998).

${ }^{20}$ M. Yamamoto, K. Sudoh, and H. Iwasaki, Surf. Sci. 601, 1255 (2007).

${ }^{21}$ R. V. Kukta, A. Peralta, and D. Kouris, Phys. Rev. Lett. 88, 186102 (2002).

${ }^{22}$ G. Ehrlich and F. G. Hudda, J. Chem. Phys. 44, 1039 (1966).

${ }^{23}$ R. L. Schwoebel and E. J. Shipsey, J. Appl. Phys. 37, 3682 (1966).

${ }^{24}$ H.-C. Jeong and E. D. Williams, Surf. Sci. Rep. 34, 171 (1999).

${ }^{25}$ W. W. Pai, J. S. Ozcomert, N. C. Bartelt, T. L. Einstein, and J. E. Reutt-Robey, Surf. Sci. 307-309, 747 (1994).

${ }^{26}$ D. Margetis, P.-W. Fok, M. J. Aziz, and H. A. Stone, Phys. Rev. Lett. 97, 096102 (2006).

${ }^{27}$ P.-W. Fok, Ph.D. thesis, Massachusetts Institute of Technology, 2006.

${ }^{28}$ A. Rettori and J. Villain, J. Phys. (France) 49, 257 (1988).

${ }^{29}$ M. Ozdemir and A. Zangwill, Phys. Rev. B 42, 5013 (1990).

${ }^{30}$ H. Spohn, J. Phys. (France) 3, 69 (1993).

${ }^{31}$ H. P. Bonzel and E. Preuss, Surf. Sci. 336, 209 (1995).

${ }^{32}$ M. V. Ramana Murty, Phys. Rev. B 62, 17004 (2000).

${ }^{33}$ J. Hager and H. Spohn, Surf. Sci. 324, 365 (1995).

${ }^{34}$ H. P. Bonzel and W. W. Mullins, Surf. Sci. 350, 285 (1996).
${ }^{35}$ M. E. Keeffe, C. C. Umbach, and J. M. Blakely, J. Phys. Chem. Solids 55, 965 (1994).

${ }^{36}$ J. Erlebacher, M. J. Aziz, E. Chason, M. B. Sinclair, and J. A. Floro, Phys. Rev. Lett. 84, 5800 (2000).

${ }^{37}$ E. S. Fu, D.-J. Liu, M. D. Johnson, J. D. Weeks, and E. D. Williams, Surf. Sci. 385, 259 (1997).

${ }^{38}$ P.-W. Fok, R. R. Rosales, and D. Margetis, Phys. Rev. B 76, 033408 (2007).

${ }^{39}$ G. B. Whitham, Linear and Nonlinear Waves (Wiley, New York, 1974).

${ }^{40}$ M. R. Evans, R. Juhász, and L. Santen, Phys. Rev. E 68, 026117 (2003).

${ }^{41}$ R. Haberman, Mathematical Models: Mechanical Vibrations, Population Dynamics and Traffic Flow: An Introduction to Applied Mathematics (Prentice-Hall, Englewood Cliffs, NJ, 1977).

${ }^{42}$ Y. Homma, H. Hibino, T. Ogino, and N. Aizawa, Phys. Rev. B 55, R10237 (1997).

${ }^{43}$ C. Ratsch and A. Zangwill, Appl. Phys. Lett. 63, 2348 (1993).

${ }^{44}$ H. P. Bonzel, E. Preuss, and B. Steffen, Appl. Phys. A: Solids Surf. 35, 1 (1984).

${ }^{45}$ E. D. Williams, Surf. Sci. 299-300, 502 (1994). 